MIT-CTP-2705

UG-FT-86/98

hep-ph/9806451

June 1998

\title{
Techniques for one-loop calculations in constrained differential renormalization
}

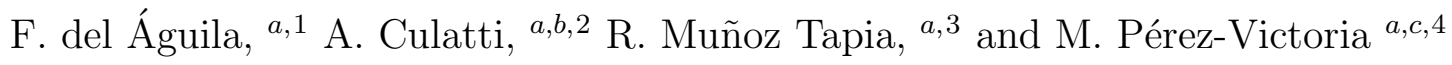 \\ ${ }^{a}$ Dpto. de Física Teórica y del Cosmos, Universidad de Granada, \\ 18071 Granada, Spain \\ ${ }^{b}$ Dip. di Fisica, Università di Padova, 35131 Padova, Italy \\ ${ }^{c}$ Center for Theoretical Physics, Laboratory for Nuclear Science and \\ Department of Physics, Massachusetts Institute of Technology, \\ Cambridge, MA 02139, USA
}

\begin{abstract}
We describe in detail the constrained procedure of differential renormalization and develop the techniques required for one-loop calculations. As an illustration we renormalize Scalar QED and show that the two-, three- and four-point Ward identities are automatically satisfied.
\end{abstract}

\section{Introduction}

Differential renormalization (DR) [ [ ] is a method of renormalization in coordinate space that yields directly finite Green functions without intermediate regularization or explicit

\footnotetext{
${ }^{1}$ e-mail: faguila@goliat.ugr.es

2e-mail: culatti@mvxpd5.pd.infn.it

${ }^{3}$ e-mail: rmt@ugr.es

${ }^{4}$ e-mail: mpv@ugr.es
} 
counterterms. It has proved to be quite simple and powerful in a number of applications [2] (see also Refs. [3, 4, 5] for formal developments). Standard DR manipulates singular objects as if they were well-defined, expresses them in terms of simple singular functions, and substitutes these by their renormalized value. The renormalized functions contain arbitrary dimensionful constants which play the role of renormalization scales and carry all the ambiguity inherent to the formal manipulation of singular expressions. In symmetric theories, these scales can be adjusted in such a way that the Ward identities for renormalized Green functions are satisfied. For practical purposes, however, one would prefer a procedure that directly rendered symmetric expressions, without having to impose the Ward identities after each calculation.

Such a procedure, constrained differential renormalization (CDR), was introduced in Ref. [6] at the one-loop level. The idea is to impose that the renormalized expressions be compatible with a minimal set of consistent formal manipulations (rules). As a result, the ambiguities and arbitrary renormalization scales of DR are fixed and the resulting renormalized Green functions automatically preserve Ward identities. This was explicitly shown in Ref. [6] for a variety of cases in abelian gauge theories. Supersymmetry was also preserved in the calculation of supergravity corrections to the anomalous magnetic moment of a charged lepton [7, 8]. The method also works for nonabelian gauge theories, as shown in Ref. [9]. The extension of the constrained method to higher orders is more involved and needs further study. At any rate, it requires a good understanding and systematization of the one-loop order.

The purpose of this paper is to describe CDR in detail and introduce the techniques required for one-loop calculations in any renormalizable theory in four dimensions. As an example, we renormalize (to one loop) Scalar QED, which contains all possible kinds of diagrams and derivative structures that appear in renormalizable theories. The plan of the paper is the following. Section 2 contains the procedure and rules of CDR. We first motivate them taking as a guiding example the vertex Ward identity in QED. Then we discuss how to expand any one-loop Feynman graph in a complete set of basic functions and we give the renormalization rules. Finally, the rules are used to determine the renormalization of the singular basic functions. The reader interested in practical calculations only needs the tables in this section. The full derivation is given in Appendix A. In Section 3 we apply the method to Scalar QED. We renormalize the two-, three- and four-point 1PI Green functions and verify that they satisfy the Ward identities. Section 4 is devoted to conclusions. In Appendix B we collect the Fourier transforms of CDR expressions. An efficient computer code performing all operations automatically (in momentum space) is available [10].

\section{Constrained differential renormalization}

In general, the bare expressions of Feynman diagrams in coordinate space are too singular at coincident points to behave as tempered distributions. The role of renormalization is to consistently replace these "singular" expressions by "regular" ones. To carry out this program, we first reduce each Feynman graph to a sum of (singular) 
basic funtions and their derivatives, and then define the renormalized diagram as the corresponding sum of the renormalized basic functions. CDR is essentially a set of rules for manipulating the singular expressions and fixing the renormalization of a complete set of basic functions. These rules are chosen in such a way that Ward identities are respected. We specify the rules below, after motivating them with a simple example. Then, we define the (one-loop) basic functions and apply the rules to renormalize them.

\subsection{Motivation of the rules}

Ward identities among Green functions are derived from the symmetries of the action using general properties of Quantum Field Theory, as gathered in Schwinger's action principle [11]. These properties are only formal for bare Green functions because they involve ill-defined expressions. Regularization and renormalization can invalidate them, breaking the symmetries at the quantum level. In absence of anomalies, the symmetry can be restored adding adequate finite local counterterms, as a consequence of the quantum action principle [12, 13]. However, in real calculations it is preferable to have an invariant procedure rendering directly symmetric expressions. This is the case of dimensional regularization with minimal substraction in gauge theories 14, 15. In perturbation theory, the fulfilment of the Ward identities (and of the action principle, in general) relies heavily on the following property [15, 16]: the application of the kinetic differential operator to the propagator corresponding to some line in a Feynman graph is equivalent to the contraction of the line to a point. In other words, the fact that the free propagators are Green functions of the corresponding quadratic lagrangian must not be spoiled by the renormalization procedure. Actually, the corresponding proof for gauge theories in Ref. [15] is based on the fact that this property holds for the dimensionally regulated diagrams. We shall see that the propagator equations also play a central role in CDR. Although no explicit regulator is available in DR, one can impose simple conditions on the renormalized expressions that guarantee the validity of the propagator equations and of other formal identities. To see what kind of constraints one must impose, let us consider the case of the vertex Ward identity in massless QED,

$$
\left(\partial_{\mu}^{x}-\partial_{\mu}^{y}\right) V_{\mu}(x, y)=i e[\Sigma(x) \delta(y)-\Sigma(y) \delta(x)]
$$

where $V_{\mu}$ is the 1PI photon-electron-electron vertex, $\Sigma$ is the electron self-energy and $x \equiv x_{1}-x_{2}, y \equiv x_{2}-x_{3}$, with $x_{1,2,3}$ the external points $\left(x_{2}\right.$ being the point attached to the photon)円. For bare functions this Ward identity is easy to derive formally at one loop. Using the Feynman rules in Ref. [17] (we work in euclidean space and in the

\footnotetext{
${ }^{1}$ For convenience when treating the general case, we use in this paper the variable $y$ with a minus sign with respect to the one in Refs. [6, 8, 8, 17.
} 
Feynman gauge),

$$
\begin{aligned}
\left(\partial_{\mu}^{x}-\partial_{\mu}^{y}\right) V_{\mu}(x, y)= & \left(\partial_{\mu}^{x}-\partial_{\mu}^{y}\right)\left[(-i e)^{3} \gamma_{\alpha} \not \partial^{x} \Delta(x) \gamma_{\mu} \not \partial^{y} \Delta(y) \gamma_{\alpha} \Delta(x+y)\right] \\
= & i e^{3}\left[\gamma_{\alpha} \not^{x} \not \partial^{x} \Delta(x) \not \partial^{y} \Delta(y) \gamma_{\alpha} \Delta(x+y)\right. \\
& -\gamma_{\alpha} \not^{x} \Delta(x) \not \partial^{y} \partial^{y} \Delta(y) \gamma_{\alpha} \Delta(x+y) \\
& \left.+\gamma_{\alpha} \not \partial^{x} \Delta(x) \gamma_{\mu} \partial^{y} \Delta(y) \gamma_{\alpha}\left(\partial_{\mu}^{x}-\partial_{\mu}^{y}\right) \Delta(x+y)\right] \\
= & i e^{3}\left[-\delta(x) \gamma_{\alpha} \not \partial^{y} \Delta(y) \gamma_{\alpha} \Delta(x+y)\right. \\
& \left.+\delta(y) \gamma_{\alpha} \not \partial^{x} \Delta(x) \gamma_{\alpha} \Delta(x+y)\right] \\
= & i e[-\delta(x) \Sigma(y)+\delta(y) \Sigma(x)]
\end{aligned}
$$

where $\Delta(x)=\frac{1}{4 \pi^{2}} \frac{1}{x^{2}}$ is the massless Feynman propagator, $\partial_{\mu}^{x}$ stands for $\frac{\partial}{\partial x_{\mu}}$ and $\square=\partial_{\mu} \partial_{\mu}$. The manipulations used in Eq. (2.2) are only formal because we are dealing with singular expressions (at $x=y$ ). We have first performed the linear change of variables described above, and used the Leibnitz rule to make the external derivatives act on individual propagators. Then we have used the propagator equation for a massless fermion (which is equivalent to replacing $\not \partial \not \partial$ by $\square$ and using the scalar propagator equation, $\square \Delta=-\delta)$. The symmetry of $\Delta(x+y)$ under the interchange of $x$ and $y$ makes $\left(\partial_{\mu}^{x}-\partial_{\mu}^{y}\right) \Delta(x+y)$ vanish; finally, the definition of the Dirac delta function justifies the last equality. When these ill-defined expressions are regularized or replaced by finite ones (renormalized), some of these manipulations may not be justified. For example, if the fermion propagator were naïvely regulated à la Pauli-Villars, $S^{P V}(x)=\not \partial^{x}\left(\Delta(x)-\Delta_{\Lambda}(x)\right)$, with $\Delta_{\Lambda}$ the Feynman propagator of mass $\Lambda$, the propagator equation would be modified: $\not \partial S^{P V}=-\Lambda^{2} \Delta_{\Lambda}(x)$, and the third equality in Eq. (2.2) would no longer hold. Other two examples studied in the DR literature are the regulators introduced in Refs. [4] and [1], respectively. In the former, the modification of the propagator invalidates the propagator equation; in the latter the short distance expansion invalidates the change of variables and the direct use of the delta function?. Our aim is to find a DR scheme automatically preserving the Ward identities for renormalized amplitudes. One may require the whole Eq. (2.1) to be satisfied by the corresponding renormalized expressions, as we essentially did in Ref. [7] to relate diagrams with different topology. However this has to be improved because diagrams are expected to be renormalized independently of the particular combination in which they appear in the Ward identities. Instead, we demand that the manipulations performed to derive Eq. (2.2) be always valid for renormalized expressions. In other words, we require that DR commutes with this kind of manipulations. In what follows we show how this can be done consistently at one loop. Indeed, a few rules will be sufficient to fix the ambiguities of standard DR and to ensure the validity of the Ward identities at the same time.

\subsection{Rules for constrained differential renormalization}

We distinguish two kinds of rules. The first ones simply state that renormalization commutes with algebraic identities that allow to perform "straightforward" manipu-

\footnotetext{
${ }^{2}$ Both methods were adequate to study the relation between DR and the usual counterterm approach, although the spirit of DR is precisely to avoid the use of such regulators.
} 
lations, like sums of terms, Dirac algebra (in four dimensions), or application of the Leibnitz rule. With these manipulations one can express any one-loop Feynman diagram in terms of a set of basic functions that will be defined in the next subsection. The rules of the second kind extend well-defined identities of distribution theory to more singular expressions. They will be used to determine the renormalization of the basic functions. Let us enumerate them (the first two rules are essentially the prescriptions of the method of DR) [6]:

1. Differential reduction: singular expressions are substituted by derivatives of regular ones. This can be done in two steps:

(a) Functions with singular behaviour worse than logarithmic $\left(\sim x^{-4}\right)$ are reduced to derivatives of logarithmically singular functions without introducing extra dimensionful constants.

(b) Logarithmically singular functions are written as derivatives of regular functions. The usual DR identity [1]

$$
\left[\frac{1}{x^{4}}\right]^{R}=-\frac{1}{4} \square \frac{\log x^{2} M^{2}}{x^{2}}
$$

is sufficient to one loop. This identity also applies to massive expressions when they are expanded in the mass. Eq. (2.3) introduces the unique dimensionful constant of the whole process, $M$, which has dimensions of mass and plays the role of the renormalization group scale.

2. Formal integration by parts: derivatives act formally by parts on test functions. In particular,

$$
[\partial F]^{R}=\partial F^{R}
$$

where $F$ is an arbitrary function and $R$ stands for renormalized.

3. Delta function renormalization rule:

$$
\left[F\left(x, x_{1}, \ldots, x_{n}\right) \delta(x-y)\right]^{R}=\left[F\left(x, x_{1}, \ldots, x_{n}\right)\right]^{R} \delta(x-y) .
$$

4. The general validity of the propagator equation:

$$
\left[F\left(x, x_{1}, \ldots, x_{n}\right)\left(\square^{x}-m^{2}\right) \Delta_{m}(x)\right]^{R}=\left[F\left(x, x_{1}, \ldots, x_{n}\right)(-\delta(x))\right]^{R},
$$

where $\Delta_{m}(x)=\frac{1}{4 \pi^{2}} \frac{m K_{1}(m x)}{x}$ and $K_{1}$ is a modified Bessel function [18].

Rule 1, combined with rule 2, reduces the "degree of singularity", connecting singular and regular expressions. Forbidding the introduction of dimensionful scales outside logarithms, we completely fix the scheme. The last three rules are valid mathematical identities among tempered distributions when applied to a well-behaved enough function $F$. The rules formally extend their range of applicability to arbitrary functions. They require that these identities commute with the process of renormalization. 
Rule 2 is essential to make sense of rule 1, for otherwise the right-hand-side of it would not be a well-defined distribution. Rule 1 connects functions with different number of propagators and has actually been applied in the literature to reduce three-point functions to two-point functions, thus allowing the use of the DR identity (2.3). As we shall see in the next subsection, it has further important implications. Note that the massive propagator equation (2.6) carries the necessary information for the propagators appearing in usual theories. Indeed, the corresponding equation for a massless scalar propagator is just the limit $m \rightarrow 0$ of Eq. (2.6), the equation for a fermionic propagator follows from it, after the use of $\not \partial \not \partial=\square$, and the equation for the propagator of a gauge boson in a general covariant gauge can be derived from the first two terms of the mass expansion of Eq. (2.6) (see Ref. [6]).

All these rules allow to renormalize any one-loop Feynman graph. Other possible manipulations can be incompatible with them and introduce ambiguities. One can still perform them as long as one keeps track of the arbitrary local terms they introduce. These local terms can then be fixed imposing consistency with the renormalization rules. In particular, two dangerous operations are explicit differentiation (which is not well-defined at coincident points) $]^{3}$ and index contraction [6, 17], as we shall see explicitly later on. These two manipulations do not commute in general with CDR.

The actual procedure of renormalization involves two steps: (1) express a Feynman diagram in terms of basic functions and (2) replace the basic functions by their renormalized value. The renormalization of the basic functions is done once and for all and does not depend on the particular calculation. This is the subject of the next subsection. Let us now comment on the first step. At one loop, a Feynman graph in coordinate space contains products of propagators with differential operators acting on some of them and, possibly, delta functions. It can also include constant objects like group factors, gamma matrices, the metric and the Levi-Civita tensor. To evaluate the diagram, we first make a convenient change of variables (see below). Then, we perform all the (Dirac) algebra. In particular, we do all possible contractions. This is a necessary prescription because index contraction does not commute with CDR. This prescription leads to universal renormalized basic functions. Finally, the Leibnitz rule is used to reorder the derivatives in each term, so that they act either on the whole product or on the last propagator. Symmetries among the space-time points can also be safely used as, for example, in $F(x, y) \partial_{\mu}^{y} \Delta(x+y)=F(x, y) \partial_{\mu}^{x} \Delta(x+y)$.

\subsection{Renormalization of basic functions}

We shall assume that a Feynman-like gauge is chosen for the gauge fields, so that their propagators are proportional to the scalar Feynman propagator. The formalism can be directly extended to general covariant gauges, as was indicated in Ref. [6]. Up to delta functions, any one-loop 1PI graph is a linear combination of products of scalar propagators $\Delta_{m_{i}}\left(x_{i}-x_{i+1}\right)$, with differential operators $\mathcal{O}^{x_{i}}$ acting on them. All points $x_{i}$ are external and appear in a cyclic way. Therefore, using the Leibnitz rule, each

\footnotetext{
${ }^{3}$ Taking derivatives is essential to obtain the DR identities, but the corresponding ambiguities are eventually fixed by the CDR rules.
} 
term of the graph can be written (ignoring constants and delta functions) as a sum of total derivatives of the whole product of propagators, with all the internal derivatives acting on only one adequately chosen propagator:

$$
\begin{aligned}
& \mathcal{O}_{1}^{x_{1}} \Delta_{m_{1}}\left(x_{1}-x_{2}\right) \mathcal{O}_{2}^{x_{2}} \Delta_{m_{2}}\left(x_{2}-x_{3}\right) \cdots \mathcal{O}_{n}^{x_{n}} \Delta_{m_{n}}\left(x_{n}-x_{1}\right) \\
& =\mathcal{O}_{1}^{z_{1}} \Delta_{m_{1}}\left(z_{1}\right) \mathcal{O}_{2}^{z_{2}} \Delta_{m_{2}}\left(z_{2}\right) \cdots \mathcal{O}_{n}^{-z_{1}} \Delta_{m_{n}}\left(z_{1}+z_{2}+\cdots+z_{n-1}\right) \\
& =\sum_{i} \mathcal{O}_{\text {ext }(i)}\left[\Delta_{m_{1}}\left(z_{1}\right) \Delta_{m_{2}}\left(z_{2}\right) \cdots \mathcal{O}_{i n t(i)}^{z_{1}} \Delta_{m_{n}}\left(z_{1}+z_{2}+\cdots+z_{n-1}\right)\right] .
\end{aligned}
$$

In the first line we have performed a convenient change of variables: $z_{1}=x_{1}-x_{2}$, $z_{2}=x_{2}-x_{3}, \ldots, z_{n-1}=x_{n-1}-x_{n}, z_{n}=x_{n}$. This eliminates one variable $\left(z_{n}\right)$ due to translational invariance. Besides, since every $z_{i}$ appears only in one of the first $n-1$ propagators and in the last one, it is straightforward to use the Leibnitz rule to make all derivatives act on the last propagator and obtain the second equality. Because of rule 2, the renormalization of the graph reduces to renormalizing expressions of the form

$$
\begin{aligned}
& \mathrm{F}_{m_{1} m_{2} \ldots m_{n-1} m_{n}}^{(n)}[\mathcal{O}]\left(z_{1}, z_{2}, \ldots, z_{n-1}\right) \equiv \\
& \quad \Delta_{m_{1}}\left(z_{1}\right) \Delta_{m_{2}}\left(z_{2}\right) \cdots \Delta_{m_{n-1}}\left(z_{n-1}\right) \mathcal{O}^{z_{1}} \Delta_{m_{n}}\left(z_{1}+z_{2}+\cdots+z_{n-1}\right)
\end{aligned}
$$

which we call basic functions. For massless basic functions we shall suppress the mass subindices. In renormalizable theories, singular one-loop diagrams involve basic functions with at most four propagators and no more derivatives than propagators. All the singular basic functions for this class of theories (in the Feynman gauge) are displayed in Table 1. We denote A, B, T and Q the basic functions with one, two, three and four propagators, respectively. In the following we shall use $x, y, z$ to denote $z_{1,2,3}$, respectively, and assume that they are the (ordered) arguments of the basic functions unless otherwise specified (e.g., $\mathrm{T}[\square] \equiv \mathrm{T}[\square](x, y)$ ). The renormalization of these functions is carried out using the rules of CDR 田- Renormalized one- and two-point functions are gathered in Tables 2 and 3, and three- and four-point functions, in Table 4. For the reader's convenience the massless A and B functions are given in Table 2, although they can be obtained from the massive ones in Table 3, taking the appropriate limit. The expressions for $\mathrm{T}$ and $\mathrm{Q}$ functions in Table 4 apply directly for any value of the masses. The Fourier transforms are collected in Appendix B. The reader interested in applications only needs the expressions in Tables 2, 3 and 4 for coordinate space or Tables 5, 6 and 7 in Appendix B for momentum space calculations.

Note that it is important in our procedure to distinguish basic functions with contracted and uncontracted differential operators, because contraction of Lorentz indices does not in general commute with CDR. For instance, from Table 4 [6, 17],

$$
\mathrm{T}^{R}[\square]=\left[\delta_{\mu \nu} \mathrm{T}\left[\partial_{\mu} \partial_{\nu}\right]\right]^{R} \neq \delta_{\mu \nu} \mathrm{T}^{R}\left[\partial_{\mu} \partial_{\nu}\right]
$$

This justifies our prescription of contracting indices before identifying the basic functions. In the following we briefly describe how these tables have been obtained. The detailed derivation can be found in Appendix A. 


\begin{tabular}{l|c|c|c|c} 
& logarithmic & linear & quadratic & cubic \\
\hline 1 prop. & & & $\mathrm{A}_{m}[1]$ & $\mathrm{A}_{m}\left[\partial_{\mu}\right]$ \\
\hline 2 props. & $\mathrm{B}_{m_{1} m_{2}}[1]$ & $\mathrm{B}_{m_{1} m_{2}}\left[\partial_{\mu}\right]$ & $\mathrm{B}_{m_{1} m_{2}}[\square]$ & \\
& & & $\mathrm{B}_{m_{1} m_{2}}\left[\partial_{\mu} \partial_{\nu}\right]$ & \\
\hline 3 props. & $\mathrm{T}_{m_{1} m_{2} m_{3}}[\square]$ & $\mathrm{T}_{m_{1} m_{2} m_{3}}\left[\square \partial_{\mu}\right]$ & & \\
& $\mathrm{T}_{m_{1} m_{2} m_{3}}\left[\partial_{\mu} \partial_{\nu}\right]$ & $\mathrm{T}_{m_{1} m_{2} m_{3}}\left[\partial_{\mu} \partial_{\nu} \partial_{\rho}\right]$ & & \\
\hline & $\mathrm{Q}_{m_{1} m_{2} m_{3} m_{4}}[\square \square]$ & & & \\
4 props. & $\mathrm{Q}_{m_{1} m_{2} m_{3} m_{4}}\left[\square \partial_{\mu} \partial_{\nu}\right]$ & & & \\
& $\mathrm{Q}_{m_{1} m_{2} m_{3} m_{4}}\left[\partial_{\mu} \partial_{\nu} \partial_{\rho} \partial_{\sigma}\right]$ & & & \\
\hline
\end{tabular}

Table 1: Singular basic functions for renormalizable theories in four dimensions. Lines are ordered according to the number of propagators and columns according to the degree of singularity. The function $\mathrm{A}$, that appears in tadpoles, is defined as $\mathrm{A}_{m}[\mathcal{O}]=$ $\mathcal{O} \Delta_{m}(x) \delta(x)$.

In general the renormalization of basic functions proceeds in two steps. First, a differential equation is solved for non-singular points, in order to express the singular basic functions as derivatives of well-behaved functions. A useful trick in the case of complex tensor structures is to decompose them into trace and traceless parts: the former carries the leading singularity but is simpler, while the latter is less singular. Second, the arbitrary local terms are determined according to the CDR rules. Rule i is actually just an initial condition for the local terms, while the remaining rules relate the local terms of different basic functions. Basic functions with simple enough tensor structures can be directly expressed in terms of other basic functions that have been previously renormalized.

Let us consider first the renormalization of $\mathrm{B}_{m_{1} m_{2}}[1]$ and $\mathrm{A}_{m}[1]$. We start with the massless case. Direct application of rule 1 gives

$$
\mathrm{B}^{R}[1]=\frac{1}{\left(4 \pi^{2}\right)^{2}}\left[\frac{1}{x^{4}}\right]^{R}=-\frac{1}{64 \pi^{4}} \square \frac{\log x^{2} M^{2}}{x^{2}}
$$

which is the standard DR identity. The fact that $\mathrm{A}[1]=\Delta(x) \delta(x)$ is local, together with power counting, implies that the most general renormalized value of this function is of the form

$$
\mathrm{A}^{R}[1]=\left(a \square+\mu^{2}\right) \delta(x) .
$$

Rule 1 a tells us not to introduce the dimensionful constant $\mu$, so the second term in the equation above vanishes. Eq. (2.10) and $\mu=0$ in Eq. (2.11), based on rule 11, are the initial conditions of the renormalization process. This rule is not needed anymore. As it is shown in Appendix A, rule 3 implies that the first term in Eq. (2.11) must also vanish, so we have

$$
\mathrm{A}^{R}[1]=0 .
$$




$$
\begin{aligned}
& \mathrm{A}^{R}[1]=0 \\
& \mathrm{~A}^{R}\left[\partial_{\mu}\right]=0 \\
& \mathrm{~B}^{R}[1]=-\frac{1}{64 \pi^{4}} \square \frac{\log x^{2} M^{2}}{x^{2}} \\
& \mathrm{~B}^{R}\left[\partial_{\mu}\right]=\frac{1}{2} \partial_{\mu} \mathrm{B}^{R}[1] \\
& \mathrm{B}^{R}[\square]=0 \\
& \mathrm{~B}^{R}\left[\partial_{\mu} \partial_{\nu}\right]=\frac{1}{3}\left(\partial_{\mu} \partial_{\nu}-\frac{1}{4} \delta_{\mu \nu} \square\right) \mathrm{B}^{R}[1]+\frac{1}{288 \pi^{2}}\left(\partial_{\mu} \partial_{\nu}-\delta_{\mu \nu} \square\right) \delta(x)
\end{aligned}
$$

Table 2: Renormalized expressions of massless one- and two-point basic functions.

For massive basic functions we use recurrence relations among modified Bessel functions to obtain the expressions for non-singular points (see Ref. [5, 18] and Appendix C of Ref. [7]). The local terms of $\mathrm{B}_{m_{1} m_{2}}^{R}[1]$ and $\mathrm{A}_{m}^{R}[1]$ are fixed so that in the massless limit we recover $\mathrm{B}^{R}[1]$ and $\mathrm{A}^{R}[1]$, respectively. In this way we find:

$$
\begin{aligned}
\mathrm{B}_{m_{1} m_{2}}^{R}[1]= & \frac{1}{\left(4 \pi^{2}\right)^{2}}\left[\frac{m_{1} K_{1}\left(m_{1} x\right) m_{2} K_{1}\left(m_{2} x\right)}{x^{2}}\right]^{R} \\
= & \frac{1}{32 \pi^{4}}\left\{\frac{m_{1} m_{2}}{m_{1}+m_{2}}\left[\square-\left(m_{1}+m_{2}\right)^{2}\right] \frac{K_{0}\left(m_{1} x\right) K_{1}\left(m_{2} x\right)+K_{0}\left(m_{2} x\right) K_{1}\left(m_{1} x\right)}{x}\right. \\
& \left.+2 \pi^{2}\left(\log \frac{\bar{M}^{2}}{m_{1} m_{2}}+\frac{m_{1}-m_{2}}{m_{1}+m_{2}} \log \frac{m_{2}}{m_{1}}\right) \delta(x)\right\},
\end{aligned}
$$

where $\bar{M}=2 M / \gamma_{E}$ and $\gamma_{E}=1.781 \ldots$ is Euler's constant. The massive one-point function $\mathrm{A}_{m}^{R}[1]$ is determined (see Appendix $\mathrm{A}$ ) from $\mathrm{A}^{R}[1]$ and $\mathrm{B}_{m_{1} m_{2}}^{R}$ :

$$
\mathrm{A}_{m}^{R}[1]=\frac{1}{16 \pi^{2}} m^{2}\left(1-\log \frac{\bar{M}^{2}}{m^{2}}\right) \delta(x) .
$$

The renormalization of the remaining basic functions is obtained from $\mathrm{B}_{m_{1} m_{2}}^{R}[1]$ and $\mathrm{A}_{m}[1]^{R}\left(\mathrm{~B}^{R}[1]\right.$ and $\mathrm{A}^{R}[1]$ in the massless case) by recurrence relations based on rules 2 , 3 and 1 . These recurrence relations follow from the Leibnitz rule and two operations: point separation and point contraction. Point separation [7] allows to relate a generic renormalized basic function with $n$ propagators and $r$ derivatives, $\mathrm{F}_{m_{1} \ldots m_{n}}^{(n) R}\left[\mathcal{O}^{(r)}\right]$, to renormalized basic functions with $n+1$ propagators and $r, r+1$ and $r+2$ derivatives. Using the rules,

$\mathrm{F}_{m_{1} \ldots m_{n}}^{(n) R}[\mathcal{O}]\left(z_{1}, \ldots, z_{n-1}\right) \delta\left(z_{n}\right)$ 


$$
\begin{aligned}
\mathrm{A}_{m}^{R}[1] & =\frac{1}{16 \pi^{2}} m^{2}\left(1-\log \frac{\bar{M}^{2}}{m^{2}}\right) \delta(x) \\
\mathrm{A}_{m}^{R}\left[\partial_{\mu}\right]=0 & \\
\mathrm{~B}_{m_{1} m_{2}}^{R} & {[1]=\frac{1}{32 \pi^{4}}\left\{\frac{m_{1} m_{2}}{m_{1}+m_{2}}\left[\square-\left(m_{1}+m_{2}\right)^{2}\right] \frac{K_{0}\left(m_{1} x\right) K_{1}\left(m_{2} x\right)+K_{0}\left(m_{2} x\right) K_{1}\left(m_{1} x\right)}{x}\right.} \\
& \left.+2 \pi^{2}\left(\log \frac{\bar{M}^{2}}{m_{1} m_{2}}+\frac{m_{1}-m_{2}}{m_{1}+m_{2}} \log \frac{m_{2}}{m_{1}}\right) \delta(x)\right\} \\
\mathrm{B}_{m_{1} m_{2}}^{R} & {\left[\partial_{\mu}\right]=\frac{1}{2} \partial_{\mu} \mathrm{B}_{m_{1} m_{2}}^{R}[1]+\frac{1}{64 \pi^{4}}\left[m_{2}^{2} K_{0}\left(m_{2} x\right) \partial_{\mu} \frac{m_{1} K_{1}\left(m_{1} x\right)}{x}-m_{1}^{2} K_{0}\left(m_{1} x\right) \partial_{\mu} \frac{m_{2} K_{1}\left(m_{2} x\right)}{x}\right] } \\
\mathrm{B}_{m_{1} m_{2}}^{R}[ & {[\square]=m_{2}^{2} \mathrm{~B}_{m_{1} m_{2}}^{R}[1]-A_{m_{1}}^{R}[1] } \\
\mathrm{B}_{m_{1} m_{2}}^{R} & {\left[\partial_{\mu} \partial_{\nu}\right]=\frac{1}{2}\left(\partial_{\mu} \mathrm{B}_{m_{1} m_{2}}^{R}\left[\partial_{\nu}\right]-\partial_{\nu} \mathrm{B}_{m_{2} m_{1}}^{R}\left[\partial_{\mu}\right]\right)+\frac{1}{8} \delta_{\mu \nu}\left(\mathrm{B}_{m_{1} m_{2}}^{R}[\square]+\mathrm{B}_{m_{2} m_{1}}^{R}[\square]\right) } \\
& +\frac{1}{3}\left(\partial_{\mu} \partial_{\nu}-\frac{1}{4} \delta_{\mu \nu} \square\right) \mathrm{B}_{m_{1}}^{R} m_{2}[1] \\
& +\frac{1}{192 \pi^{4}}\left\{\left[m_{1}^{2} \frac{m_{1} K_{1}\left(m_{1} x\right)}{x}\left(\partial_{\mu} \partial_{\nu}-\frac{1}{4} \delta_{\mu \nu} \square\right) K_{0}\left(m_{2} x\right)\right.\right. \\
& \left.+m_{2}^{2} \frac{m_{2} K_{1}\left(m_{2} x\right)}{x}\left(\partial_{\mu} \partial_{\nu}-\frac{1}{4} \delta_{\mu \nu} \square\right) K_{0}\left(m_{1} x\right)\right] \\
& -\left[m_{1}^{2} K_{0}\left(m_{1} x\right)\left(\partial_{\mu} \partial_{\nu}-\frac{1}{4} \delta_{\mu \nu} \square\right) \frac{m_{2} K_{1}\left(m_{2} x\right)}{x}\right. \\
& \left.\left.+m_{2}^{2} K_{0}\left(m_{2} x\right)\left(\partial_{\mu} \partial_{\nu}-\frac{1}{4} \delta_{\mu \nu} \square\right) \frac{m_{1} K_{1}\left(m_{1} x\right)}{x}\right]\right\} \\
& +\frac{1}{16 \pi^{2}}\left[\frac{1}{18}\left(\partial_{\mu} \partial_{\nu}-\delta_{\mu \nu} \square\right)+\frac{1}{8}\left(m_{1}^{2}+m_{2}^{2}\right) \delta_{\mu \nu}\right] \delta(x)
\end{aligned}
$$

Table 3: Renormalized expressions of massive one- and two-point basic functions.

$$
\begin{aligned}
= & {\left[\Delta_{m_{1}}\left(z_{1}\right) \ldots \Delta_{m_{n-1}}\left(z_{n-1}\right) \mathcal{O}^{z_{1}} \Delta_{m_{n}}\left(z_{1}+\cdots+z_{n-1}\right) \delta\left(z_{n}\right)\right]^{R} } \\
= & {\left[\Delta_{m_{1}}\left(z_{1}\right) \ldots \Delta_{m_{n-1}}\left(z_{n-1}\right) \mathcal{O}^{z_{1}} \Delta_{m_{n}}\left(z_{1}+\cdots+z_{n-1}+z_{n}\right) \delta\left(z_{n}\right)\right]^{R} } \\
= & -\left[\Delta_{m_{1}}\left(z_{1}\right) \ldots \Delta_{m_{n-1}}\left(z_{n-1}\right) \mathcal{O}^{z_{1}} \Delta_{m_{n}}\left(z_{1}+\cdots+z_{n-1}+z_{n}\right)\left(\square^{z_{n}}-m_{n+1}^{2}\right) \Delta_{m_{n+1}}\left(z_{n}\right)\right]^{R} \\
= & \left(m_{n+1}^{2}-\square^{z_{n}}\right) \mathrm{F}_{m_{1} \ldots m_{n-1} m_{n+1} m_{n}}^{(n+1) R}[\mathcal{O}]\left(z_{1}, \ldots, z_{n}\right) \\
& +2 \partial_{\rho}^{z_{n}} \mathrm{~F}_{m_{1} \ldots m_{n-1} m_{n+1} m_{n}}^{(n+1) R}\left[\mathcal{O} \partial_{\rho}\right]\left(z_{1}, \ldots, z_{n}\right) \\
& -\mathrm{F}_{m_{1} \ldots m_{n-1} m_{n+1} m_{n}}^{(n+1) R}[\mathcal{O} \square]\left(z_{1}, \ldots, z_{n}\right) .
\end{aligned}
$$

On the other hand, point contraction relates a renormalized basic function with a d'alambertian and $r$ derivatives to renormalized basic functions with one less propagator and $0,1, \ldots, r$ derivatives:

$$
\begin{aligned}
& \mathrm{F}_{m_{1} \ldots m_{n+1}}^{(n+1) R}[\mathcal{O} \square]\left(z_{1}, \ldots, z_{n}\right) \\
& =\left[\Delta_{m_{1}}\left(z_{1}\right) \ldots \Delta_{m_{n}}\left(z_{n}\right) \mathcal{O}^{z_{1}} \square^{z_{1}} \Delta_{m_{n+1}}\left(z_{1}+\cdots+z_{n}\right)\right]^{R} \\
& =-\left[\Delta_{m_{1}}\left(z_{1}\right) \ldots \Delta_{m_{n}}\left(z_{n}\right) \mathcal{O}^{z_{1}}\left(\delta\left(z_{1}+\cdots+z_{n}\right)-m_{n+1}^{2} \Delta_{m_{n+1}}\left(z_{1}+\cdots+z_{n}\right)\right)\right]^{R}
\end{aligned}
$$




$$
\begin{aligned}
& \mathrm{T}_{m_{1} m_{2} m_{3}}^{R}[\square]=m_{3}^{2} \mathrm{~T}_{m_{1} m_{2} m_{3}}[1]-\mathrm{B}_{m_{1} m_{2}}^{R}[1] \delta(x+y) \\
& \mathrm{T}_{m_{1} m_{2} m_{3}}^{R}\left[\partial_{\mu} \partial_{\nu}\right]=\mathrm{T}_{m_{1} m_{2} m_{3}}\left[\partial_{\mu} \partial_{\nu}-\frac{1}{4} \delta_{\mu \nu} \square\right]+\frac{1}{4} \delta_{\mu \nu} \mathrm{T}_{m_{1} m_{2} m_{3}}^{R}[\square]-\frac{1}{128 \pi^{2}} \delta_{\mu \nu} \delta(x) \delta(y) \\
& \mathrm{T}_{m_{1} m_{2} m_{3}}^{R}\left[\square \partial_{\mu}\right]=m_{3}^{2} \mathrm{~T}_{m_{1} m_{2} m_{3}}\left[\partial_{\mu}\right]-\mathrm{B}_{m_{1} m_{2}}^{R}\left[\partial_{\mu}\right] \delta(x+y)-\partial_{\mu}^{y}\left(\mathrm{~B}_{m_{1} m_{2}}^{R}[1] \delta(x+y)\right) \\
& \mathrm{T}_{m_{1} m_{2} m_{3}}^{R}\left[\partial_{\mu} \partial_{\nu} \partial_{\rho}\right]=\mathrm{T}_{m_{1} m_{2} m_{3}}\left[\partial_{\mu} \partial_{\nu} \partial_{\rho}-\frac{1}{6}\left(\delta_{\mu \nu} \partial_{\rho}+\delta_{\mu \rho} \partial_{\nu}+\delta_{\nu \rho} \partial_{\mu}\right) \square\right] \\
& \quad+\frac{1}{6}\left(\delta_{\mu \nu} \mathrm{T}_{m_{1} m_{2} m_{3}}^{R}\left[\square \partial_{\rho}\right]+\delta_{\mu \rho} \mathrm{T}_{m_{1} m_{2} m_{3}}^{R}\left[\square \partial_{\nu}\right]+\delta_{\nu \rho} \mathrm{T}_{m_{1} m_{2} m_{3}}^{R}\left[\square \partial_{\mu}\right]\right) \\
& \quad-\frac{1}{576 \pi^{2}}\left(\delta_{\mu \nu}\left(\partial_{\rho}^{x}+\partial_{\rho}^{y}\right)+\delta_{\mu \rho}\left(\partial_{\nu}^{x}+\partial_{\nu}^{y}\right)+\delta_{\nu \rho}\left(\partial_{\mu}^{x}+\partial_{\mu}^{y}\right)\right)(\delta(x) \delta(y)) \\
& \quad \mathrm{Q}_{m_{1} m_{2} m_{3} m_{4}}^{R}[\square \square]=m_{4}^{2} \mathrm{Q}_{m_{1} m_{2} m_{3} m_{4}}[\square]-\square^{z}\left(\mathrm{~T}_{m_{1} m_{2} m_{3}}[1] \delta(x+y+z)\right) \\
& \quad-2 \partial_{\rho}^{z}\left(\mathrm{~T}_{m_{1} m_{2} m_{3}}\left[\partial_{\rho}\right] \delta(x+y+z)\right)-\mathrm{T}_{m_{1} m_{2} m_{3}}^{R}[\square] \delta(x+y+z) \\
& \mathrm{Q}_{m_{1} m_{2} m_{3} m_{4}}^{R}\left[\square \partial_{\mu} \partial_{\nu}\right]=m_{4}^{2} \mathrm{Q}_{m_{1} m_{2} m_{3} m_{4}}\left[\partial_{\mu} \partial_{\nu}\right]-\partial_{\mu}^{z} \partial_{\nu}^{z}\left(\mathrm{~T}_{m_{1} m_{2} m_{3}}[1] \delta(x+y+z)\right) \\
& \quad-\partial_{\mu}^{z}\left(\mathrm{~T}_{m_{1} m_{2} m_{3}}\left[\partial_{\nu}\right] \delta(x+y+z)\right)-\partial_{\nu}^{z}\left(\mathrm{~T}_{m_{1} m_{2} m_{3}}\left[\partial_{\mu}\right] \delta(x+y+z)\right) \\
& \quad-\mathrm{T}_{m_{1} m_{2} m_{3}}^{R}\left[\partial_{\mu} \partial_{\nu}\right] \delta(x+y+z) \\
& \quad \begin{array}{l}
\mathrm{Q}_{m_{1} m_{2} m_{3} m_{4}}^{R}\left[\partial_{\mu} \partial_{\nu} \partial_{\rho} \partial_{\sigma}\right]=\mathrm{Q}_{m_{1} m_{2} m_{3} m_{4}}\left[\partial_{\mu} \partial_{\nu} \partial_{\rho} \partial_{\sigma}-\frac{1}{24}\left(\delta_{\mu \nu} \delta_{\rho \sigma}+\delta_{\mu \rho} \delta_{\nu \sigma}+\delta_{\mu \sigma} \delta_{\nu \rho}\right) \square \square\right] \\
\quad+\frac{1}{24}\left(\delta_{\mu \nu} \delta_{\rho \sigma}+\delta_{\mu \rho} \delta_{\nu \sigma}+\delta_{\mu \sigma} \delta_{\nu \rho}\right)\left(\mathrm{Q}_{m_{1} m_{2} m_{3} m_{4}}^{R}[\square \square]+\frac{5}{96 \pi^{2}} \delta(x) \delta(y) \delta(z)\right)
\end{array}
\end{aligned}
$$

Table 4: Renormalized expressions of three- and four-point basic functions. The basic functions with traceless differential operators are directly finite.

$$
\begin{aligned}
= & \sum_{i} \mathcal{O}_{i}^{z_{n}}\left(\mathrm{~F}_{m_{1} \ldots m_{n}}^{(n) R}\left[\mathcal{O}_{i}^{\prime}\right]\left(z_{1}, \ldots, z_{n-1}\right) \delta\left(z_{1}+\cdots+z_{n}\right)\right) \\
& +m_{n+1}^{2} \mathrm{~F}_{m_{1} \ldots m_{n+1}}^{(n+1) R}[\mathcal{O}]\left(z_{1}, \ldots, z_{n}\right) .
\end{aligned}
$$

$\mathcal{O}_{i}\left(\mathcal{O}_{i}^{\prime}\right)$ is a differential operator with $r_{i}\left(r-r_{i}\right)$ derivatives, $0 \leq r_{i} \leq r$. Observe that point contraction and point separation are not inverse operations of each other due to the different delta functions in the l.h.s. of Eq. (2.15) and in the r.h.s. of Eq. (2.16). This allows to obtain non-trivial information when Eq. (2.16) is inserted in the last term of Eq. (2.15). In Appendix A we show how the combined use of the operations just described fixes the renormalization of all the singular basic functions of Table 1.

\section{Renormalization of Scalar QED}

In this section we apply the procedure of CDR to Scalar QED and verify that the Ward identities among renormalized Green functions are satisfied. The presence of derivative couplings makes Scalar QED the simplest renormalizable theory that contains all the singular basic functions in Table 1 . In this context, the techniques described above can 

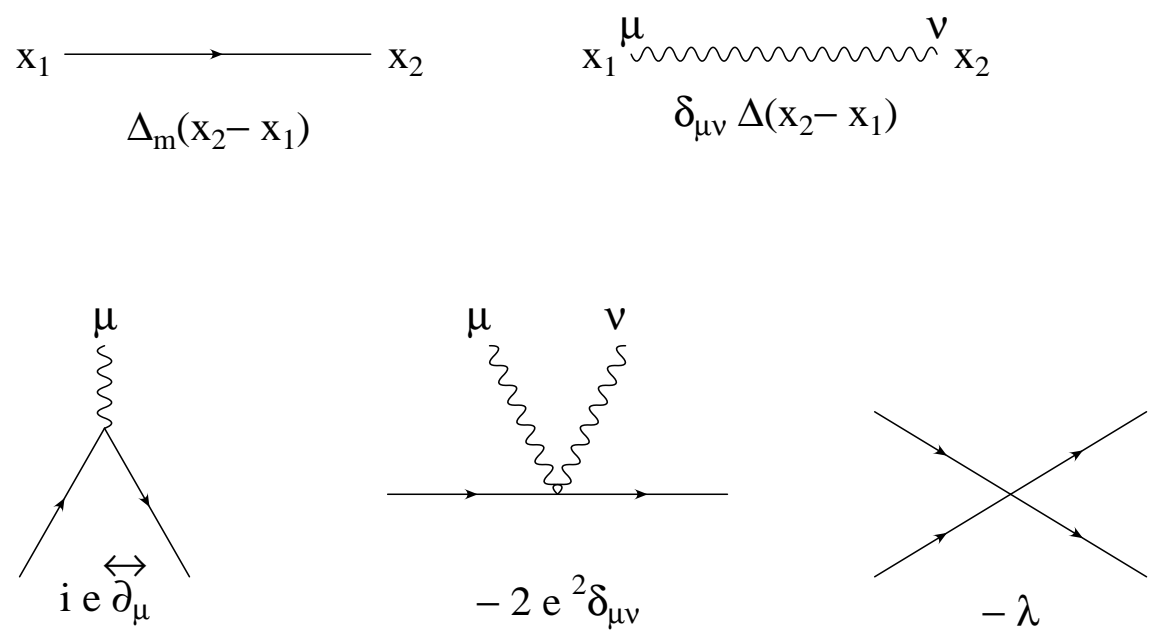

Figure 1: Feynman rules of Scalar QED. Wavy lines correspond to photons and solid lines to scalars. In the trilinear coupling, $\stackrel{\leftrightarrow}{\partial}_{\mu}=\vec{\partial}_{\mu}-\overleftarrow{\partial}_{\mu}$, with $\vec{\partial}_{\mu}\left(\overleftarrow{\partial}_{\mu}\right)$ acting on the incoming (outgoing) scalar.

be fully illustrated. We calculate to one loop the 1PI Green functions of two, three and four points, which are the only ones that require renormalization. The one-point Green functions directly vanish. In the following, after giving the lagrangian and the Feynman rules, we briefly discuss the renormalization of the six 1PI Green functions that contain singular graphs, namely the vacuum polarization, the scalar selfenergy, the photon-scalar-scalar interaction vertex, and the three kinds of four particle vertices: photon-photon, photon-scalar and scalar-scalar scattering, and the corresponding Ward identities. We also check that the one-loop $\beta$-functions and anomalous dimensions of Scalar QED are recovered.

The complete lagrangian for Scalar QED in euclidean space and in the Feynman gauge is

$$
\mathcal{L}_{E}=\frac{1}{4} F_{\mu \nu} F_{\mu \nu}+\frac{1}{2}\left(\partial_{\mu} A_{\mu}\right)^{2}+\left(\partial_{\mu}-i e A_{\mu}\right) \phi^{\dagger}\left(\partial_{\mu}+i e A_{\mu}\right) \phi+m^{2} \phi^{\dagger} \phi+\frac{\lambda}{4}\left(\phi^{\dagger} \phi\right)^{2} .
$$

The corresponding Feynman rules are depicted in Fig.1. 


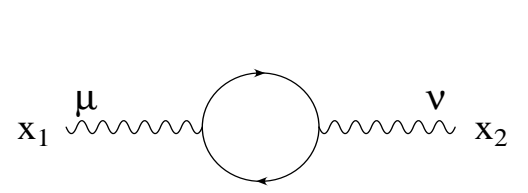

(a1)

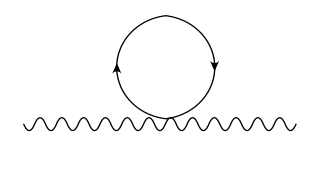

(a2)

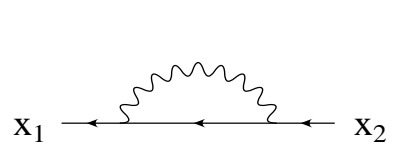

(b1)

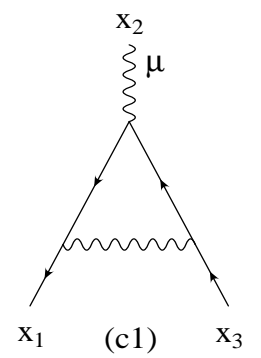

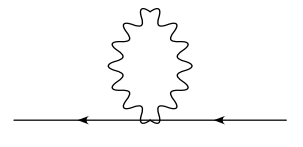

(b2)

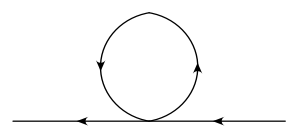

(b3)

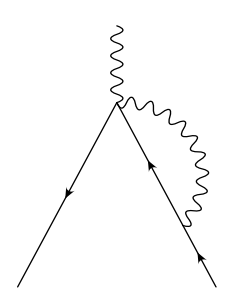

(c2)

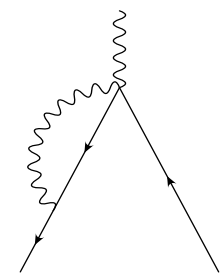

(c3)

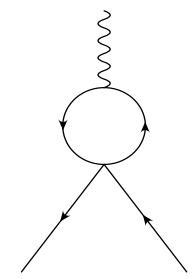

(c4)

Figure 2: Feynman diagrams contributing to the vacuum polarization (a), scalar selfenergy (b) and photon-scalar-scalar vertex (c). 


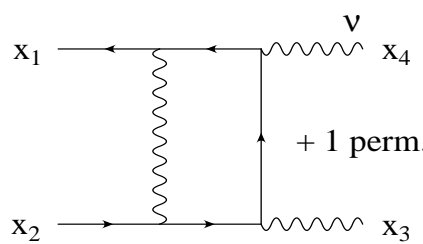

(d1)

$\mu$

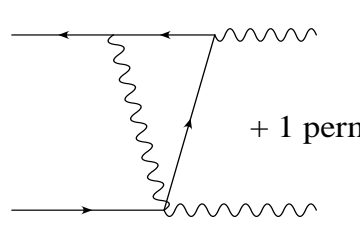

(d2)

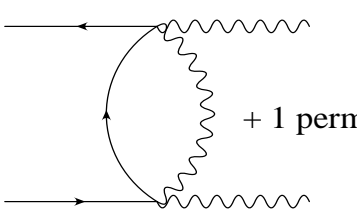

(d5) (d4)

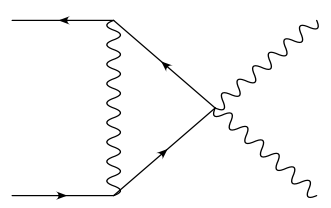

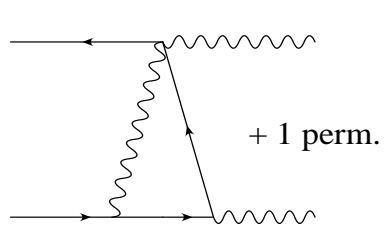

(d3)

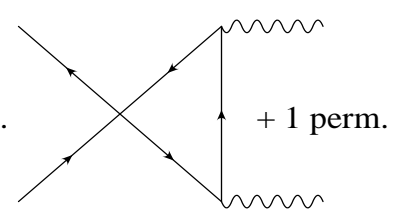

(d6)

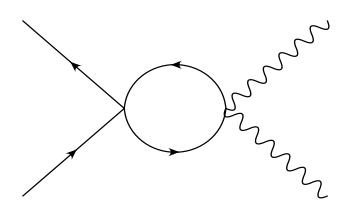

(d7)

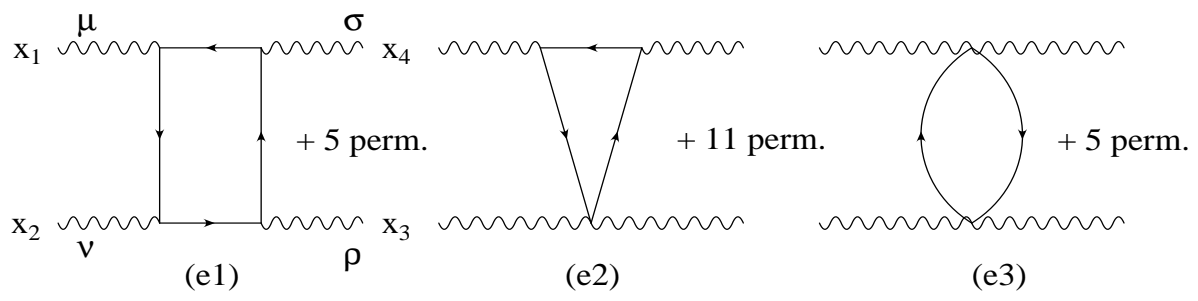

Figure 3: Feynman diagrams contributing to the photon-scalar (d) and photon-photon (e) scattering. The permutations take into account diagrams with opposite charge flow. 


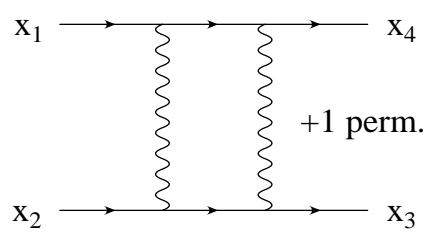

(f1)

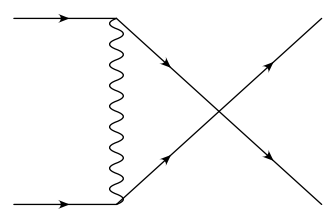

(f4)

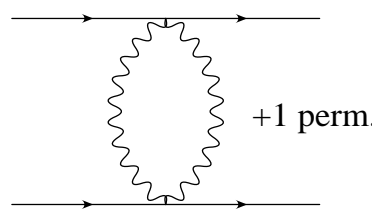

(f7)

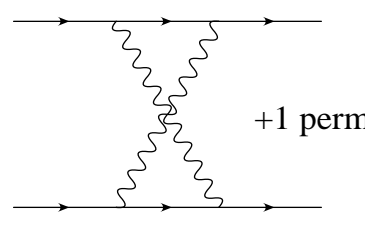

(f2)

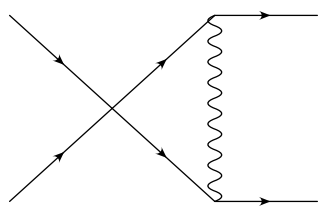

(f5)

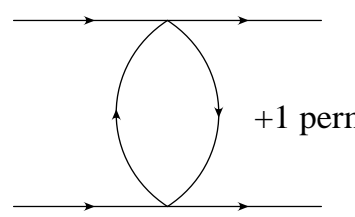

(f8)

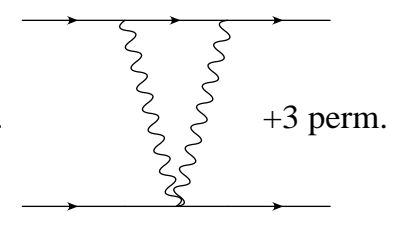

(f3)

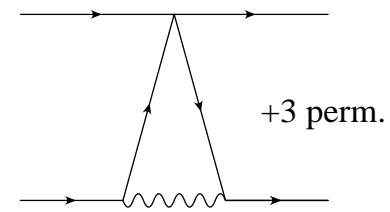

(f6)

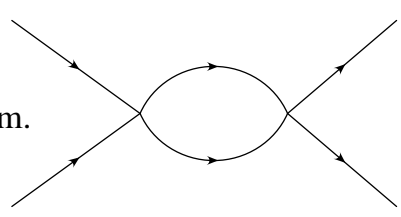

(f9)

Figure 4: Feynman diagrams contributing to the scalar-scalar scattering (f). 


\subsection{Vacuum polarization}

The two Feynman graphs contributing to the vacuum polarization, a1 and a2, are depicted in Fig. 2. Their contributions are

$$
\begin{aligned}
\Pi_{(a) \mu \nu}(x) & =-e^{2} \Delta_{m}(x) \overleftrightarrow{\partial}_{\mu} \overleftrightarrow{\partial}_{\nu} \Delta_{m}(x), \\
\Pi_{(b) \mu \nu}(x) & =-2 e^{2} \delta_{\mu \nu} \Delta_{m}(x) \delta(x),
\end{aligned}
$$

where $x=x_{1}-x_{2}$ is the coordinate difference. In the following, unless otherwise stated, we shall use the shifted variables defined in the text. In terms of basic functions the two contributions read

$$
\begin{aligned}
\Pi_{(a) \mu \nu}(x) & =-e^{2}\left(4 \mathrm{~B}_{m m}\left[\partial_{\mu} \partial_{\nu}\right]-\partial_{\mu} \partial_{\nu} \mathrm{B}_{m m}[1]\right), \\
\Pi_{(b) \mu \nu}(x) & =-2 e^{2} \delta_{\mu \nu} \mathrm{A}_{m} .
\end{aligned}
$$

Using the expressions of the renormalized functions in Table 3, the sum is [6]

$$
\begin{aligned}
\Pi_{\mu \nu}^{R}(x)= & -\frac{e^{2}}{\left(4 \pi^{2}\right)^{2}}\left(\partial_{\mu} \partial_{\nu}-\delta_{\mu \nu} \square\right)\left[\frac { 1 } { 6 } ( \square - 4 m ^ { 2 } ) \left(\frac{m K_{0}(m x) K_{1}(m x)}{x}\right.\right. \\
& \left.\left.+m^{2}\left(K_{0}^{2}(m x)-K_{1}^{2}(m x)\right)\right)+\frac{1}{3} \pi^{2}\left(\log \frac{\bar{M}^{2}}{m^{2}}-\frac{4}{3}\right) \delta(x)\right],
\end{aligned}
$$

which is transverse, as required by the Ward identity

$$
\partial_{\mu}^{x} \Pi_{\mu \nu}^{R}(x)=0 .
$$

The scale-dependent part is

$$
M \frac{\partial}{\partial M} \Pi_{\mu \nu}^{R}(x)=-\frac{e^{2}}{24 \pi^{2}}\left(\partial_{\mu} \partial_{\nu}-\delta_{\mu \nu} \square\right) \delta(x) .
$$

\subsection{Scalar selfenergy}

The one-loop scalar selfenergy is obtained from graphs b1, b2 and b3 in Fig. 2:

$$
\begin{aligned}
\Sigma_{(a)}(x)= & -e^{2}\left[\square\left(\Delta(x) \Delta_{m}(x)\right)+2 \partial_{\alpha}\left(\Delta(x) \partial_{\alpha} \Delta_{m}(x)\right)\right. \\
& \left.+\Delta(x) \square \Delta_{m}(x)\right], \\
\Sigma_{(b)}(x)= & -4 e^{2} \Delta(x) \delta(x), \\
\Sigma_{(c)}(x)= & -\lambda \Delta_{m}(x) \delta(x),
\end{aligned}
$$

where derivatives acting on the external scalars have been changed by minus derivatives acting on the whole amputated expression. In terms of basic functions, the total renormalized selfenergy is

$$
\Sigma^{R}(x)=-e^{2}\left(\square \mathrm{B}_{0 m}^{R}[1]+2 \partial_{\rho} \mathrm{B}_{0 m}^{R}\left[\partial_{\rho}\right]+\mathrm{B}_{0 m}^{R}[\square]+4 \mathrm{~A}^{R}[1]\right)-\lambda \mathrm{A}_{m}^{R}[1] .
$$

The explicit expression can be obtained using Tables 2 and 3. The scale-dependent part reads

$$
M \frac{\partial}{\partial M} \Sigma^{R}(x)=\left(-\frac{e^{2}}{8 \pi^{2}}\left(2 \square+m^{2}\right)+\frac{\lambda}{8 \pi^{2}} m^{2}\right) \delta(x) .
$$




\subsection{Photon-scalar-scalar vertex}

The contributing diagrams, c1-c4, are shown in Fig. 2. Their renormalized expressions in terms of basic functions are

$$
\begin{aligned}
V_{(a) \mu}^{R}= & -i e^{3}\left\{-2 \mathrm{~T}_{m m 0}^{R}\left[\square \partial_{\mu}\right]+\left(\partial_{\mu}^{x}+\partial_{\mu}^{y}\right) \mathrm{T}_{m m 0}^{R}[\square]+4\left(\partial_{\alpha}^{x}+\partial_{\alpha}^{y}\right) \mathrm{T}_{m m 0}^{R}\left[\partial_{\mu} \partial_{\alpha}\right]\right. \\
& -8 \partial^{x} \cdot \partial^{y} \mathrm{~T}_{m m 0}\left[\partial_{\mu}\right]-2\left(\partial_{\mu}^{x}+\partial_{\mu}^{y}\right)\left(\partial_{\alpha}^{x}+\partial_{\alpha}^{y}\right) \mathrm{T}_{m m 0}\left[\partial_{\alpha}\right] \\
& \left.+4\left(\partial_{\mu}^{x}+\partial_{\mu}^{y}\right) \partial^{x} \cdot \partial^{y} \mathrm{~T}[1]\right\} \\
V_{(b) \mu}^{R}= & -2 i e^{3}\left\{\partial_{\mu}^{y} \mathrm{~B}_{0 m}^{R}[1](y)+\mathrm{B}_{0 m}^{R}\left[\partial_{\mu}\right](y)\right\} \delta(x), \\
V_{(c) \mu}^{R}= & -2 i e^{3}\left\{\partial_{\mu}^{x} \mathrm{~B}_{0 m}^{R}[1](x)+\mathrm{B}_{0 m}^{R}\left[\partial_{\mu}\right](x)\right\} \delta(y), \\
V_{(d) \mu}^{R}= & 0 .
\end{aligned}
$$

Graph c4, which is proportional to $\lambda$, vanishes directly because of charge conjugation. The renormalized vertex results from the sum of Eqs. (3.12-3.14) and the use of Tables 3 and 4. The scale-dependent part is

$$
M \frac{\partial}{\partial M} V_{\mu}^{R}(x, y)=-i \frac{e^{3}}{4 \pi^{2}}\left(\partial_{\mu}^{x}+\partial_{\mu}^{y}\right) \delta(x) \delta(y) .
$$

The renormalized vertex function and selfenergy fulfil the Ward identity

$$
\left(\partial_{\mu}^{x}-\partial_{\mu}^{y}\right) V_{\mu}^{R}(x, y)=i e\left(\Sigma^{R}(x) \delta(y)-\Sigma(y)^{R} \delta(x)\right),
$$

analogous to Eq. (2.1) in QED. This can be checked integrating with any test function $\phi(x, y)$. In particular, $\phi(x, y)=e^{i p \cdot x}$ leads to simple expressions for both sides of the identity [17]. Observe that the part of the Ward identity proportional to $\lambda$ (coming from diagrams b3 and c4) is trivially satisfied, for both sides vanish. In general, the diagrams proportional to $\lambda$ form a gauge invariant subset.

\subsection{Four-point functions}

The diagrams contributing to the photon-scalar $\left(V_{\mu \nu}\right), \mathrm{d} 1-\mathrm{d} 7$, and photon-photon $\left(V_{\mu \nu \rho \sigma}\right)$, e1-e3, and to the scalar-scalar $(V)$, f1-f9, scattering are depicted in Figs. 3 and 4, respectively. As in previous cases, the renormalized Green functions are obtained expanding in basic functions and substituting these by their renormalized expressions in Tables 24. The final results are too lengthy to be reported here. We have calculated them with a symbolic program based on Mathematica. The scale-dependent parts are readily obtained. For the photon-photon-scalar-scalar vertex we have

$$
M \frac{\partial}{\partial M} V_{\mu \nu}^{R}(x, y, z)=\delta_{\mu \nu} \frac{e^{4}}{2 \pi^{2}} \delta(x) \delta(y) \delta(z) .
$$

Notice that there is no $\lambda$-dependent part. Actually, Eqs. (3.16) and (3.18) follow from gauge invariance and Eq. (3.6). The scale-dependent part of the four-photon vertex 
cancels when all diagams are summed, as required by renormalizability. Finally, the scale-dependent part of the four-scalar vertex is

$$
M \frac{\partial}{\partial M} V(x, y, z)=\frac{1}{16 \pi^{2}}\left(24 e^{4}-4 e^{2} \lambda+5 \lambda^{2}\right) \delta(x) \delta(y) \delta(z) .
$$

Observe that there is a $\lambda$-independent piece, reflecting the well-known fact that a quartic scalar self-coupling is needed in the lagrangian for multiplicative renormalizability. The Ward identities for the four-point functions are more transparent in terms of the original external points:

$$
\begin{aligned}
& \partial_{\mu}^{x_{3}} V_{\mu \nu}^{R}\left(x_{1}, x_{2}, x_{3}, x_{4}\right)=i e V_{\nu}^{R}\left(x_{1}, x_{4}, x_{2}\right)\left(\delta\left(x_{1}-x_{3}\right)-\delta\left(x_{2}-x_{3}\right)\right), \\
& \partial_{\mu}^{x_{1}} V_{\mu \nu \rho \sigma}^{R}\left(x_{1}, x_{2}, x_{3}, x_{4}\right)=0,
\end{aligned}
$$

and the identities related to Eqs. (3.20) and (3.21) by Bose symmetry. There is no Ward identity relating $V$ to other singular Green functions. In terms of the shifted variables, Eqs. (3.20) and (3.21) read

$$
\begin{aligned}
& \left(\partial_{\mu}^{z}-\partial_{\mu}^{y}\right) V_{\mu \nu}^{R}(x, y, z)=i e V_{\nu}^{R}(x+y+z,-y-z)[\delta(x+y)-\delta(y)], \\
& \partial_{\mu}^{x} V_{\mu \nu \rho \sigma}^{R}(x, y, z)=0 .
\end{aligned}
$$

The scale-dependent parts of $V_{\mu \nu}^{R}, V_{\mu}^{R}$ and $V_{\mu \nu \rho \sigma}^{R}$ trivially satisfy Eqs. (3.22) and (3.23). The merit of CDR is to give also the correct scale-independent parts of the Ward identities. We have checked numerically that this is indeed the case, supplementing the program used for the calculation of the renormalized Green functions with the $F F$ package [19] ( $F F$ works in dimensional regularization, but we have only used it for finite functions).

\subsection{Renormalization group}

To conclude this section we compute the first order anomalous dimensions and $\beta$ functions. The renormalization group equation for any renormalized amplitude reads

$$
\left(M \frac{\partial}{\partial M}+\beta_{e} \frac{\partial}{\partial e}+\beta_{\lambda} \frac{\partial}{\partial \lambda}+\gamma_{m} m \frac{\partial}{\partial m}-n_{\phi} \gamma_{\phi}-n_{A} \gamma_{A}\right) \Gamma^{R\left(n_{\phi}, n_{A}\right)}=0,
$$

where $\gamma_{\phi, A}$ are the anomalous dimensions of the corresponding fields and $\Gamma^{R\left(n_{\phi}, n_{A}\right)}$ is the 1PI renormalized amplitude of $n_{\phi}$ scalar and $n_{A}$ photon fields ${ }^{4}$. The tree level part of the $\Gamma$ functions can be read off from the Feynman rules in Fig. 1 and the scaledependence of the one-loop amplitudes is given in Eqs. (3.6), (3.11), (3.16), (3.18) and (3.19). Using the renormalization group equations (3.24) for the relevant amplitudes one obtains

$$
\gamma_{A}=\frac{e^{2}}{48 \pi^{2}}
$$

\footnotetext{
${ }^{4}$ The only effect of the gauge parameter dependence is to consistently cancel the tree-level longitudinal term in the equation for the vacuum polarization.
} 


$$
\begin{aligned}
\gamma_{\phi} & =-\frac{e^{2}}{8 \pi^{2}}, \\
\gamma_{m} & =\frac{\lambda-3 e^{2}}{16 \pi^{2}}, \\
\beta_{e} & =\frac{e^{3}}{48 \pi^{2}}, \\
\beta_{\lambda} & =\frac{24 e^{4}-12 e^{2} \lambda+5 \lambda^{2}}{16 \pi^{2}} .
\end{aligned}
$$

Notice that $\beta_{e}=e \gamma_{A}$, as dictated by gauge invariance.

\section{Conclusions}

Constrained differential renormalization is a scheme of differential renormalization which does not introduce the ambiguities inherent to formal manipulations of singular expressions. At the moment it has only been developed at the one-loop level, although in principle it could be extended to higher orders. Such extension looks rather involved and is under study. In this paper we provide the techniques and explicit expressions that allow to perform any one-loop calculation in a four dimensional renormalizable theory (in the Feynman gauge) with this method. The whole procedure has been implemented in a computer package that performs all operations automatically in momentum space. This package is available and its description can be found in Ref. [10]. Constrained differential renormalization respects the (one-loop) Ward identities of gauge invariance. Here, we have shown it for Scalar QED, which contains all the singular functions appearing in renormalizable theories. In Ref. [6] it was verified that the Ward identities in QED are satisfied and the correct triangular anomaly recovered (a more complete discussion of anomalies will be presented elsewhere). That non-abelian gauge invariance is preserved is explicitly shown in Ref. [9]. The method also preserves supersymmetry in the calculation of the supergravity corrections to the $g-2$ of a charged lepton [7, 8].

\section{Acknowledgements}

We thank T. Hahn for providing a helpful package linking Mathematica and FF. The pictures have been made with FeynDiag. MPV thanks D.Z. Freedman for discussions and MIT for its hospitality. This work has been supported by CICYT, under contract number AEN96-1672, and by Junta de Andalucía, FQM101. RMT and MPV thank Ministerio de Educación y Cultura for financial support. 


\section{A Appendix}

In this appendix we derive the renormalized expressions of all the singular basic functions appearing in renormalizable theories. Essentially, we use the techniques of point separation and point contraction, although in the simplest cases some intermediate steps can be avoided. For simplicity, we deal first with massless functions and then discuss the minor modifications of the process needed for the massive case.

\section{A.1 Massless basic functions}

Let us start with the one-point basic functions. We saw in the text that rule 1 a implied $\mathrm{A}^{R}[1]=a \square \delta(x)$, with $a$ arbitrary in principle. Consider now the expression $\mathrm{A}^{R}[1] \delta(y)$. Using rule 3 ,

$$
\begin{aligned}
\mathrm{A}^{R}[1](x) \delta(y) & =[\Delta(x) \delta(x)]^{R} \delta(y)=[\Delta(x) \delta(x) \delta(x+y)]^{R} \\
& =\mathrm{A}^{R}[1](x) \delta(x+y) .
\end{aligned}
$$

Integration on $x$ leads to the equation

$$
\delta(y) \int \mathrm{d}^{4} x \mathrm{~A}^{R}[1](x)=\mathrm{A}^{R}[1](y),
$$

which, using rule 2, requires $a=0$. Therefore,

$$
\mathrm{A}^{R}[1]=0 .
$$

Similarly, locality and power counting imply that $A^{R}\left[\partial_{\mu}\right]=\left[\partial_{\mu} \Delta(x) \delta(x)\right]^{R}$ is of the form

$$
\mathrm{A}^{R}\left[\partial_{\mu}\right]=\left(a^{\prime} \square+\mu^{\prime 2}\right) \partial_{\mu} \delta(x),
$$

and the same argument used before implies $a^{\prime}=\mu^{\prime}=0$, and hence

$$
\mathrm{A}^{R}\left[\partial_{\mu}\right]=0 .
$$

So, the massless one-point basic functions are zero in CDR. (The same results are obtained with the general form of point separation and point contraction.)

Consider now the two-point basic functions. $\mathrm{B}^{R}[1]$ was already given in the text. $\mathrm{B}^{R}\left[\partial_{\mu}\right]$ is easily obtained using the Leibnitz rule and the fact that the two propagators involved are identical:

$$
\mathrm{B}^{R}\left[\partial_{\mu}\right]=\left[\Delta(x) \partial_{\mu} \Delta(x)\right]^{R}=\partial_{\mu}[\Delta(x) \Delta(x)]^{R}-\left[\partial_{\mu} \Delta(x) \Delta(x)\right]^{R}
$$

and then

$$
\mathrm{B}^{R}\left[\partial_{\mu}\right]=\frac{1}{2} \partial_{\mu}\left[(\Delta(x))^{2}\right]^{R}=\frac{1}{2} \partial_{\mu} \mathrm{B}^{R}[1]
$$

$\mathrm{B}^{R}[\square]$ is directly obtained from $\mathrm{A}^{R}[1]$ using rule 4 :

$$
\mathrm{B}^{R}[\square]=[\Delta(x) \square \Delta(x)]^{R}=-[\Delta(x) \delta(x)]^{R}=-\mathrm{A}^{R}[1]=0 .
$$


This is the simplest example of point contraction. The renormalization of $\mathrm{B}\left[\partial_{\mu} \partial_{\nu}\right]$ is more involved. First, we notice that its most general renormalized expression is

$$
\mathrm{B}^{R}\left[\partial_{\mu} \partial_{\nu}\right]=\frac{1}{3}\left(\partial_{\mu} \partial_{\nu}-\frac{1}{4} \delta_{\mu \nu} \square\right) \mathrm{B}^{R}[1]+\frac{1}{16 \pi^{2}}\left[f \partial_{\mu} \partial_{\nu}+\delta_{\mu \nu}\left(g \square+\mu^{\prime \prime 2}\right)\right] \delta(x),
$$

where the first term results from solving a differential equation at $x \neq 0$ and $f, g$ and $\mu^{\prime \prime}$ are arbitrary coefficients with dimension 0, 0 and 2, respectively, parametrizing the ambiguity in the local terms 5 . Second, applying rule to the delta function in $\mathrm{A}\left[\partial_{\mu}\right]$ we obtain

$$
0=\mathrm{A}^{R}\left[\partial_{\mu}\right]=-\partial_{\mu} B^{R}[\square]+\mathrm{B}^{R}\left[\partial_{\mu} \square\right]=\mathrm{B}^{R}\left[\partial_{\mu} \square\right]
$$

and using the Leibnitz rule to move the derivatives to the first (identical) propagator and Eqs. A.7) and (A.8),

$$
\mathrm{B}^{R}\left[\partial_{\mu} \square\right]=-\frac{1}{2} \partial_{\mu} \square \mathrm{B}^{R}[1]+2 \partial_{\rho} \mathrm{B}^{R}\left[\partial_{\mu} \partial_{\rho}\right]-\mathrm{B}^{R}\left[\partial_{\mu} \square\right] .
$$

Inserting Eq. (A.10) into Eq. (A.11), we find the consistency equation

$$
-\frac{1}{2} \square \partial_{\mu} \mathrm{B}^{R}[1]+2 \partial_{\rho} \mathrm{B}^{R}\left[\partial_{\mu} \partial_{\rho}\right]=0 .
$$

If we substitute Eq. (A.9) into Eq. (A.12) we find $\mu^{\prime \prime}=0$ and $g=-f$. For the moment, $f$ remains arbitrary. It will be fixed from the relation between $\mathrm{B}^{R}\left[\partial_{\mu} \partial_{\nu}\right]$ and $\mathrm{T}^{R}\left[\partial_{\mu} \partial_{\nu} \partial_{\rho}\right]$ later on.

The renormalization of three-point basic functions containing one d'alambertian is directly obtained via point contraction, which relates them to two-point functions already renormalized:

$$
\begin{aligned}
\mathrm{T}^{R}[\square]= & -\mathrm{B}^{R}[1] \delta(x+y), \\
\mathrm{T}^{R}\left[\square \partial_{\mu}\right]= & -\partial_{\mu}^{y}\left(\mathrm{~B}^{R}[1](x) \delta(x+y)\right)-\mathrm{B}^{R}\left[\partial_{\mu}\right](x) \delta(x+y)= \\
& -\frac{1}{2}\left(\partial_{\mu}^{x}+\partial_{\mu}^{y}\right)\left(\mathrm{B}^{R}[1] \delta(x+y)\right) .
\end{aligned}
$$

For $\mathrm{T}$ functions with more complex index structure we apply point separation to the corresponding B function with one less derivative. $\mathrm{T}^{R}\left[\partial_{\mu} \partial_{\nu}\right]$ can be decomposed into a traceless and a trace part, plus a possible ambiguous local term (symmetric under $\mu \leftrightarrow \nu$ and $x \leftrightarrow y$ and with the correct dimension) [6:

$$
\mathrm{T}^{R}\left[\partial_{\mu} \partial_{\nu}\right]=\mathrm{T}\left[\partial_{\mu} \partial_{\nu}-\frac{1}{4} \delta_{\mu \nu} \square\right]+\frac{1}{4} \delta_{\mu \nu} \mathrm{T}^{R}[\square]+\frac{1}{64 \pi^{2}} b \delta_{\mu \nu} \delta(x) \delta(y) .
$$

The traceless part is finite and the trace has already been renormalized, so we just have to fix the dimensionless coefficient $b$. Separating points,

$$
\mathrm{B}^{R}\left[\partial_{\mu}\right](x) \delta(y)=-\square^{y} \mathrm{~T}\left[\partial_{\mu}\right]+2 \partial_{\rho}^{y} \mathrm{~T}^{R}\left[\partial_{\mu} \partial_{\rho}\right]-\mathrm{T}^{R}\left[\square \partial_{\mu}\right]
$$

\footnotetext{
${ }^{5}$ The dimensionful coefficient $\mu^{\prime \prime}$ is forbidden from rule 1a, but we shall instead use recurrence relations and the initial condition $\mathrm{A}^{R}[1]=0$ to prove that it vanishes, thus showing that rule 1a is consistent with the other rules.
} 
The simplest way to solve this equation for $b$ is to integrate on $x$ (see Ref. [6]). Integration on the "separated" point, $y$, leads to a tautology (this is a general fact). We obtain the value $b=-\frac{1}{2}$. The same process can be applied to $\mathrm{T}^{R}\left[\partial_{\mu} \partial_{\nu} \partial_{\rho}\right]$. Its trace-traceless decomposition is

$$
\begin{aligned}
\mathrm{T}^{R}\left[\partial_{\mu} \partial_{\nu} \partial_{\rho}\right]= & \mathrm{T}\left[\partial_{\mu} \partial_{\nu} \partial_{\rho}-\frac{1}{6}\left(\delta_{\mu \nu} \partial_{\rho}+\delta_{\mu \rho} \partial_{\nu}+\delta_{\nu \rho} \partial_{\mu}\right) \square\right] \\
& +\frac{1}{12}\left(\delta_{\mu \nu}\left(\partial_{\rho}^{x}+\partial_{\rho}^{y}\right)+\delta_{\mu \rho}\left(\partial_{\nu}^{x}+\partial_{\nu}^{y}\right)+\delta_{\nu \rho}\left(\partial_{\mu}^{x}+\partial_{\mu}^{y}\right)\right) \\
& \times\left(-\mathrm{B}^{R}[1] \delta(x+y)+\frac{1}{16 \pi^{2}} c \delta(x) \delta(y)\right)
\end{aligned}
$$

where we have used Eq. A.14). Point separation of $\mathrm{B}^{R}\left[\partial_{\mu} \partial_{\nu}\right]$, followed by point contraction of the $\mathrm{T}$ functions with one d'alambertian, leads to

$$
\begin{aligned}
\mathrm{B}^{R}\left[\partial_{\mu} \partial_{\nu}\right](x) \delta(y)= & -\square^{y} \mathrm{~T}^{R}\left[\partial_{\mu} \partial_{\nu}\right]+2 \partial_{\rho}^{y} \mathrm{~T}^{R}\left[\partial_{\mu} \partial_{\nu} \partial_{\rho}\right] \\
& +\left(\partial_{\mu}^{x} \partial_{\nu}^{y}+\partial_{\mu}^{y} \partial_{\nu}^{x}\right)\left(\mathrm{B}^{R}[1](x) \delta(x+y)\right) \\
& +\mathrm{B}^{R}\left[\partial_{\mu} \partial_{\nu}\right](x) \delta(x+y) .
\end{aligned}
$$

Integrating on $x$ after inserting Eqs. A.15) and (A.17), we get

$$
\begin{aligned}
0= & -\square^{y} \int \mathrm{d}^{4} x \mathrm{~T}\left[\partial_{\mu} \partial_{\nu}-\frac{1}{4} \delta_{\mu \nu} \square\right]+2 \partial_{\rho}^{y} \int \mathrm{d}^{4} x \mathrm{~T}\left[\partial_{\mu} \partial_{\nu} \partial_{\rho}-\frac{1}{6}\left(\delta_{\mu \nu} \partial_{\rho}+\delta_{\mu \rho} \partial_{\nu}+\delta_{\nu \rho} \partial_{\mu}\right) \square\right] \\
& \left.+\frac{1}{16 \pi^{2}}\left[\left(\frac{1}{8}+\frac{c}{6}-f\right) \delta_{\mu \nu} \square^{y}+\left(\frac{c}{3}+f\right) \partial_{\mu}^{y} \partial_{\nu}^{y}\right)\right] \delta(y),
\end{aligned}
$$

which provides two equations (one for each tensor structure) that determine the coefficients $f$ and $c$. Calculating the (finite) integrals we find $f=\frac{1}{18}$ and $c=-\frac{1}{3}$.

For four-point basic functions, which are at most logarithmically singular, the situation is similar. Q functions with at least one d'alambertian are directly contracted into T functions:

$$
\begin{aligned}
\mathrm{Q}^{R}[\square \square]= & -\square^{z}(\mathrm{~T}[1] \delta(x+y+z))-2 \partial_{\rho}^{z}\left(\mathrm{~T}\left[\partial_{\rho}\right](x, y) \delta(x+y+z)\right) \\
& -\mathrm{T}^{R}[\square](x, y) \delta(x+y+z), \\
\mathrm{Q}^{R}\left[\square \partial_{\mu} \partial_{\nu}\right]= & -\partial_{\mu}^{z} \partial_{\nu}^{z}(\mathrm{~T}[1] \delta(x+y+z))-\partial_{\mu}^{z}\left(\mathrm{~T}\left[\partial_{\nu}\right](x, y) \delta(x+y+z)\right) \\
& -\partial_{\nu}^{z}\left(\mathrm{~T}\left[\partial_{\mu}\right](x, y) \delta(x+y+z)\right) \\
& -\mathrm{T}^{R}\left[\partial_{\mu} \partial_{\nu}\right](x, y) \delta(x+y+z) .
\end{aligned}
$$

Finally, the renormalization of $\mathrm{Q}\left[\partial_{\mu} \partial_{\nu} \partial_{\rho} \partial_{\sigma}\right]$ requires again the combined use of point separation and point contraction. The trace-traceless decomposition is not unique in this case. The simplest possibility is

$$
\begin{aligned}
\mathrm{Q}^{R}\left[\partial_{\mu} \partial_{\nu} \partial_{\rho} \partial_{\sigma}\right]= & \mathrm{Q}\left[\partial_{\mu} \partial_{\nu} \partial_{\rho} \partial_{\sigma}-\frac{1}{24}\left(\delta_{\mu \nu} \delta_{\rho \sigma}+\delta_{\mu \rho} \delta_{\nu \sigma}+\delta_{\mu \sigma} \delta_{\nu \rho}\right) \square \square\right] \\
& +\frac{1}{24}\left(\delta_{\mu \nu} \delta_{\rho \sigma}+\delta_{\mu \rho} \delta_{\nu \sigma}+\delta_{\mu \sigma} \delta_{\nu \rho}\right) \\
& \times\left(\mathrm{Q}^{R}[\square \square]+\frac{1}{16 \pi^{2}} d \delta(x) \delta(y) \delta(z)\right)
\end{aligned}
$$


Point separation of $\mathrm{T}^{R}\left[\partial_{\mu} \partial_{\nu} \partial_{\rho}\right]$ gives

$$
\mathrm{T}^{R}\left[\partial_{\mu} \partial_{\nu} \partial_{\rho}\right](x, y) \delta(z)=-\square^{z} \mathrm{Q}\left[\partial_{\mu} \partial_{\nu} \partial_{\rho}\right]+2 \partial_{\sigma}^{z} \mathrm{Q}^{R}\left[\partial_{\mu} \partial_{\nu} \partial_{\rho} \partial_{\sigma}\right]-\mathrm{Q}^{R}\left[\square \partial_{\mu} \partial_{\nu} \partial_{\rho}\right],
$$

and point contraction of $\mathrm{Q}^{R}\left[\square \partial_{\mu} \partial_{\nu} \partial_{\rho}\right]$ plus integration on $x, y$ give the equation

$$
\begin{aligned}
0= & -\square^{z} \int \mathrm{d}^{4} x \mathrm{~d}^{4} y \mathrm{Q}\left[\partial_{\mu} \partial_{\nu} \partial_{\rho}\right]+2 \partial_{\sigma}^{z} \int \mathrm{d}^{4} x \mathrm{~d}^{4} y \mathrm{Q}^{R}\left[\partial_{\mu} \partial_{\nu} \partial_{\rho} \partial_{\sigma}\right] \\
& +\int \mathrm{d}^{4} y \mathrm{~T}^{R}\left[\partial_{\mu} \partial_{\nu} \partial_{\rho}\right](y, z) .
\end{aligned}
$$

Inserting Eq. (A.23) and the expression obtained for $\mathrm{T}^{R}\left[\partial_{\mu} \partial_{\nu} \partial_{\rho}\right]$, and calculating the integrals, we find $d=\frac{5}{6}$, what completes the renormalization of $\mathrm{Q}\left[\partial_{\mu} \partial_{\nu} \partial_{\rho} \partial_{\sigma}\right]$.

\section{A.2 Massive basic functions}

The massive case is analogous to the massless one. The degree of the leading singularities is not changed by the inclusion of masses. Point separation and point contraction are used in the same manner, although now the resulting expressions contain additional terms proportional to the masses (see Eqs (2.15) and (2.16)). In order to use (when possible) symmetrically the Leibnitz rule, we decompose the basic functions into two pieces, one symmetric in the masses - that is treated as in the massless case - and one antisymmetric - that is less singular.

Let us illustrate the new features of the general massive case. The renormalization of $\mathrm{A}_{m}[1]=\Delta_{m}(x) \delta(x)$, is a bit more involved than that of $\mathrm{A}[1]$. The renormalized expression must be of the form

$$
\mathrm{A}_{m}^{R}[1]=\left(\tilde{a} \square+\tilde{\mu}^{2}\right) \delta(x) .
$$

The same argument used for $\mathrm{A}^{R}[1]$ forbids the term proportional to $\square \delta(x)$. On the other hand, using rule 1 and moving derivatives to the massive propagator we get

$$
\begin{aligned}
\mathrm{A}_{m}^{R}[1] & =-\left[\Delta_{m}(x) \square \Delta(x)\right]^{R} \\
& =-\square \mathrm{B}_{0 m}^{R}[1]+2 \partial_{\rho} \mathrm{B}_{0 m}^{R}\left[\partial_{\rho}\right]+\mathrm{A}^{R}[1]-m^{2} \mathrm{~B}_{0 m}^{R}[1] .
\end{aligned}
$$

Then, integrating this equality (with rule 2),

$$
\tilde{\mu}^{2}=-m^{2} \int \mathrm{d}^{4} x \mathrm{~B}_{0 m}^{R}[1] .
$$

So, we only have to integrate Eq. (2.13) and take the limit $m_{1} \rightarrow 0$. (The integrals of expressions containing modified Bessel functions are easily performed with the techniques described in Appendix C of Ref. [7].) The result is given in Eq. (2.14). Observe that $\tilde{\mu}$ is proportional to $m$, as it should for consistency with rule 1 a.

$\mathrm{A}_{m}^{R}\left[\partial_{\mu}\right]$ is renormalized analogously to $\mathrm{A}^{R}\left[\partial_{\mu}\right] . \mathrm{B}_{m_{1} m_{2}}^{R}[1]$ is given in the text, whereas $\mathrm{B}_{m_{1} m_{2}}^{R}\left[\partial_{\mu}\right]$ is obtained generalizing Eq. (A.7) with the decomposition mentioned above:

$$
\mathrm{B}_{m_{1} m_{2}}^{R}\left[\partial_{\mu}\right]=
$$




$$
\begin{aligned}
& \frac{1}{2}\left(\mathrm{~B}_{m_{1} m_{2}}^{R}\left[\partial_{\mu}\right]+\mathrm{B}_{m_{2} m_{1}}^{R}\left[\partial_{\mu}\right]\right)+\frac{1}{2}\left(\mathrm{~B}_{m_{1} m_{2}}\left[\partial_{\mu}\right]-\mathrm{B}_{m_{2} m_{1}}\left[\partial_{\mu}\right]\right) \\
= & \frac{1}{2} \partial_{\mu} \mathrm{B}_{m_{1} m_{2}}^{R}[1] \\
& +\frac{1}{64 \pi^{4}}\left[m_{2}^{2} K_{0}\left(m_{2} x\right) \partial_{\mu} \frac{K_{1}\left(m_{1} x\right)}{x}-m_{1}^{2} K_{0}\left(m_{1} x\right) \partial_{\mu} \frac{K_{1}\left(m_{2} x\right)}{x}\right] .
\end{aligned}
$$

On the other hand, $\mathrm{B}_{m_{1} m_{2}}[\square]$ as well as $\mathrm{T}$ and $\mathrm{Q}$ functions are renormalized following the massless case but including the terms proportional to the masses, e.g.:

$$
\mathrm{B}_{m_{1} m_{2}}^{R}[\square]=m_{2}^{2} \mathrm{~B}_{m_{1} m_{2}}^{R}[1]-\mathrm{A}_{m_{1}}^{R}[1],
$$

to be compared to Eq. (A.8). Finally, $\mathrm{B}_{m_{1} m_{2}}\left[\partial_{\mu} \partial_{\nu}\right]$ requires some work to solve the differential equation. Afterwards, the local terms are fixed analogously to the $\mathrm{B}\left[\partial_{\mu} \partial_{\nu}\right]$ case. The final result is given in Table 3 .

\section{B Appendix}

In this appendix we give all the ingredients needed to obtain the momentum space expressions of Green functions renormalized with CDR. Since the renormalized functions are well-defined distributions, they admit a finite Fourier transform without any regulator. The Fourier transform of a distribution $f\left(x_{1}, \ldots, x_{n}\right)$, where $x_{i}$ are fourdimensional variables, is

$$
\hat{f}\left(p_{1}, \ldots, p_{n}\right)=\int \mathrm{d}^{4} x_{1} \ldots \mathrm{d}^{4} x_{n} e^{i x_{1} \cdot p_{1}} \ldots e^{i x_{n} \cdot p_{n}} f\left(x_{1}, x_{2}, \ldots, x_{n}\right) .
$$

Due to rule 2, total derivatives directly yield

$$
\partial_{\mu}^{x_{j}} \rightarrow-i p_{j \mu} .
$$

Delta functions give rise to reduced Fourier transforms, with linear combinations of the original momenta as arguments. For instance,

$$
f\left(x_{1}, \ldots, x_{n-1}\right) \delta\left(x_{n-1}+x_{n}\right) \rightarrow \hat{f}\left(p_{1}, \ldots, p_{n-1}-p_{n}\right) .
$$

Therefore, one only needs the Fourier transforms of the renormalized basic functions.

We shall perform Fourier transforms in the shifted variables $z_{i}$. The Fourier transform of basic functions that are directly finite without renormalization is simply a convolution in momentum space [17:

$$
\begin{aligned}
& \hat{\mathrm{F}}_{m_{1} \ldots m_{n}}^{(n)}[\mathcal{O}]\left(p_{1}, \ldots, p_{n-1}\right) \\
& =\int \frac{\mathrm{d}^{4} k}{(2 \pi)^{4}} \frac{\hat{\mathcal{O}}(k)}{\left[k^{2}+m_{n}^{2}\right]\left[\left(k-p_{1}\right)^{2}+m_{1}^{2}\right] \ldots\left[\left(k-p_{n-1}\right)^{2}+m_{n-1}^{2}\right]} \\
& \equiv \mathrm{I}_{m_{n} m_{1} \ldots m_{n-1}}^{(n)}[\hat{\mathcal{O}}]\left(p_{1}, \ldots, p_{n-1}\right) .
\end{aligned}
$$


The integrals $\mathrm{I}^{(n)}$ appear in usual one-loop calculations in momentum space and can be calculated with standard techniques.

To Fourier transform the renormalized basic functions of Tables 2, 3 and 4 we only need (besides Eq. (B.4)) the following integrals:

$$
\begin{aligned}
\int \mathrm{d}^{4} x e^{i x \cdot p} \frac{\log x^{2} M^{2}}{x^{2}} & =\frac{4 \pi^{2}}{p^{2}} \log \bar{M}^{2} p^{2}, \\
\int \mathrm{d}^{4} x e^{i x \cdot p} \frac{m K_{1}(m x)}{x} & =4 \pi^{2} \frac{1}{p^{2}+m^{2}}, \\
\int \mathrm{d}^{4} x e^{i x \cdot p} K_{0}(m x) & =8 \pi^{2} \frac{1}{\left(p^{2}+m^{2}\right)^{2}}, \\
\int \mathrm{d}^{4} x e^{i x \cdot p} K_{0}\left(m_{1} x\right) \mathcal{O} \frac{m_{2} K_{1}\left(m_{2} x\right)}{x} & =32 \pi^{4} \mathrm{I}_{m_{2} m_{1} m_{1}}^{(3)}[\hat{\mathcal{O}}](p, p), \\
\int \mathrm{d}^{4} x e^{i x \cdot p} \frac{m_{1} K_{1}\left(m_{1} x\right)}{x} \mathcal{O} K_{0}\left(m_{2} x\right) & =32 \pi^{4} \mathrm{I}_{m_{2} m_{2} m_{1}}^{(3)}[\hat{\mathcal{O}}](0, p) .
\end{aligned}
$$

The Fourier transforms involving modified Bessel functions are easily obtained using recurrence relations among them [7, 18]. The recurrence relations include delta functions to make them valid at the origin.

In Tables 5, 6 and 7 we collect the Fourier transforms of the basic functions in Tables 2, 3 and 4, respectively. These basic functions in (euclidean) momentum space can be directly used in momentum space calculations (see Ref. [10]).

$$
\begin{aligned}
& \hat{\mathrm{A}}^{R}[1]=0 \\
& \hat{\mathrm{A}}^{R}\left[\partial_{\mu}\right]=0 \\
& \hat{\mathrm{B}}^{R}[1]=\frac{1}{16 \pi^{2}} \log \frac{\bar{M}^{2}}{p^{2}-i \epsilon} \\
& \hat{\mathrm{B}}^{R}\left[\partial_{\mu}\right]=-\frac{1}{2} i p_{\mu} \hat{\mathrm{B}}^{R}[1] \\
& \hat{\mathrm{B}}^{R}[\square]=0 \\
& \hat{\mathrm{B}}^{R}\left[\partial_{\mu} \partial_{\nu}\right]=-\frac{1}{3}\left(p_{\mu} p_{\nu}-\frac{1}{4} p^{2} \delta_{\mu \nu}\right) \hat{\mathrm{B}}^{R}[1]-\frac{1}{288 \pi^{2}}\left(p_{\mu} p_{\nu}-p^{2} \delta_{\mu \nu}\right)
\end{aligned}
$$

Table 5: Fourier transforms of massless one- and two-point renormalized basic functions. The small imaginary part in the logarithms here and in the next table, allows to analitically continue these formulae into the Minkowsky region $\left(p^{2}<0\right)$. 


$$
\begin{aligned}
& \begin{aligned}
& \hat{\mathrm{A}}_{m}^{R}[1]=\frac{1}{16 \pi^{2}} m^{2}\left(1-\log \frac{\bar{M}^{2}}{m^{2}}\right) \\
& \hat{\mathrm{A}}_{m}^{R}\left[\partial_{\mu}\right]=0 \\
& \hat{\mathrm{B}}_{m_{1} m_{2}}^{R}[1]=\frac{1}{16 \pi^{2}}\left\{\frac{m_{2}^{2}-m_{1}^{2}}{p^{2}} \log \frac{m_{2}}{m_{1}}+\log \frac{\bar{M}^{2}}{m_{1} m_{2}}+C_{m_{1} m_{2}}(p)\right\} \\
& \hat{\mathrm{B}}_{m_{1} m_{2}}^{R}\left[\partial_{\mu}\right]=-\frac{1}{2} i p_{\mu}\left[\hat{\mathrm{B}}_{m_{1} m_{2}}^{R}[1]+\frac{1}{16 \pi^{2}} \frac{1}{p^{2}}\left(m_{1}^{2}-m_{2}^{2}+\log \frac{m_{1}}{m_{2}}\left(m_{1}^{2}+m_{2}^{2}+\frac{\left(m_{1}^{2}-m_{2}^{2}\right)^{2}}{p^{2}}\right)\right.\right. \\
&\left.\left.\quad+\left(m_{1}^{2}-m_{2}^{2}\right) C_{m_{1} m_{2}}(p)\right)\right] \\
& \hat{\mathrm{B}}_{m_{1} m_{2}}^{R}[\square]=m_{2}^{2} \hat{\mathrm{B}}_{m_{1} m_{2}}^{R}[1]-\hat{\mathrm{A}}_{m_{1}}^{R}[1] \\
& \hat{\mathrm{B}}_{m_{1} m_{2}}^{R}\left[\partial_{\mu} \partial_{\nu}\right]=-\frac{i}{2}\left(p_{\mu} \hat{\mathrm{B}}_{m_{1} m_{2}}^{R}\left[\partial_{\nu}\right]-p_{\nu} \hat{\mathrm{B}}_{m_{2} m_{1}}^{R}\left[\partial_{\mu}\right]\right)+\frac{1}{8} \delta_{\mu \nu}\left(\hat{\mathrm{B}}_{m_{1} m_{2}}^{R}[\square]+\hat{\mathrm{B}}_{m_{2} m_{1}}^{R}[\square]\right) \\
& \quad-\frac{1}{3}\left(p_{\mu} p_{\nu}-\frac{1}{4} \delta_{\mu \nu} p^{2}\right) \hat{\mathrm{B}}_{m_{1} m_{2}}^{R}[1] \\
& \quad-\frac{1}{6}\left\{\left[m_{1}^{2} \mathrm{I}_{m_{2} m_{2} m_{1}}^{(3)}\left[k_{\mu} k_{\nu}-\frac{1}{4} \delta_{\mu \nu} k^{2}\right](0, p)+m_{2}^{2} \mathrm{I}_{m_{1} m_{1} m_{2}}^{(3)}\left[k_{\mu} k_{\nu}-\frac{1}{4} \delta_{\mu \nu} k^{2}\right](0, p)\right]\right. \\
&\left.\quad-\left[m_{1}^{2} \mathrm{I}_{m_{2} m_{1} m_{1}}^{(3)}\left[k_{\mu} k_{\nu}-\frac{1}{4} \delta_{\mu \nu} k^{2}\right](p, p)+m_{2}^{2} \mathrm{I}_{m_{1} m_{2} m_{2}}^{(3)}\left[k_{\mu} k_{\nu}-\frac{1}{4} \delta_{\mu \nu} k^{2}\right](p, p)\right]\right\} \\
& \quad-\frac{1}{16 \pi^{2}}\left[\frac{1}{18}\left(p_{\mu} p_{\nu}-\delta_{\mu \nu} p^{2}\right)-\frac{1}{8}\left(m_{1}^{2}+m_{2}^{2}\right) \delta_{\mu \nu}\right]
\end{aligned}
\end{aligned}
$$

where

$$
\begin{aligned}
& C_{m_{1} m_{2}}(p)=-\frac{\lambda^{1 / 2}}{2 p^{2}}\left(\log \left(p^{2}+m_{1}^{2}+m_{2}^{2}+\lambda^{1 / 2}+i \epsilon\right)-\log \left(p^{2}+m_{1}^{2}+m_{2}^{2}-\lambda^{1 / 2}-i \epsilon\right)\right), \\
& \lambda=\left(p^{2}+\left(m_{1}+m_{2}\right)^{2}\right)\left(p^{2}+\left(m_{1}-m_{2}\right)^{2}\right)
\end{aligned}
$$

Table 6: Fourier transforms of massive one- and two-point renormalized basic functions. The integrals $\mathrm{I}^{(3)}$ are defined in the text. The momentum $k$ is the integration variable. 


$$
\begin{aligned}
& \hat{\mathrm{T}}_{m_{1} m_{2} m_{3}}^{R}[\square]=m_{3}^{2} \mathrm{I}_{m_{1} m_{2} m_{3}}^{(3)}[1]\left(p_{x}, p_{y}\right)-\hat{\mathrm{B}}_{m_{1} m_{2}}^{R}[1]\left(p_{x}-p_{y}\right) \\
& \hat{\mathrm{T}}_{m_{1} m_{2} m_{3}}^{R}\left[\partial_{\mu} \partial_{\nu}\right]=-\mathrm{I}_{m_{1} m_{2} m_{3}}^{(3)}\left[k_{\mu} k_{\nu}-\frac{1}{4} \delta_{\mu \nu} k^{2}\right]\left(p_{x}, p_{y}\right)+\frac{1}{4} \delta_{\mu \nu} \hat{\mathrm{T}}_{m_{1} m_{2} m_{3}}^{R}[\square]\left(p_{x}, p_{y}\right)-\frac{1}{128 \pi^{2}} \delta_{\mu \nu} \\
& \hat{\mathrm{T}}_{m_{1} m_{2} m_{3}}^{R}\left[\square \partial_{\mu}\right]=-i m_{3}^{2} \mathrm{I}_{m_{1} m_{2} m_{3}}^{(3)}\left[k_{\mu}\right]\left(p_{x}, p_{y}\right)-\hat{\mathrm{B}}_{m_{1} m_{2}}^{R}\left[\partial_{\mu}\right]\left(p_{x}-p_{y}\right)+i p_{y \mu} \hat{\mathrm{B}}_{m_{1} m_{2}}^{R}[1]\left(p_{x}-p_{y}\right) \\
& \hat{\mathrm{T}}_{m_{1} m_{2} m_{3}}^{R}\left[\partial_{\mu} \partial_{\nu} \partial_{\rho}\right]=i \mathrm{I}_{m_{1} m_{2} m_{3}}^{(3)}\left[k_{\mu} k_{\nu} k_{\rho}-\frac{1}{6}\left(\delta_{\mu \nu} k_{\rho}+\delta_{\mu \rho} k_{\nu}+\delta_{\nu \rho} k_{\mu}\right) k^{2}\right]\left(p_{x}, p_{y}\right) \\
& +\frac{1}{6}\left(\delta_{\mu \nu} \hat{\mathrm{T}}_{m_{1} m_{2} m_{3}}^{R}\left[\square \partial_{\rho}\right]\left(p_{x}, p_{y}\right)+\delta_{\mu \rho} \hat{\mathrm{T}}_{m_{1} m_{2} m_{3}}^{R}\left[\square \partial_{\nu}\right]\left(p_{x}, p_{y}\right)\right. \\
& \left.+\delta_{\nu \rho} \hat{\mathrm{T}}_{m_{1} m_{2} m_{3}}^{R}\left[\square \partial_{\mu}\right]\left(p_{x}, p_{y}\right)\right) \\
& +\frac{i}{576 \pi^{2}}\left(\delta_{\mu \nu}\left(p_{x \rho}+p_{y \rho}\right)+\delta_{\mu \rho}\left(p_{x \nu}+p_{y \nu}\right)+\delta_{\nu \rho}\left(p_{x \mu}+p_{y \mu}\right)\right) \\
& \hat{\mathrm{Q}}_{m_{1} m_{2} m_{3} m_{4}}^{R}[\square \square]=-m_{4}^{2} \mathrm{I}_{m_{1} m_{2} m_{3} m_{4}}^{(4)}\left[k^{2}\right]\left(p_{x}, p_{y}, p_{z}\right)+p_{z}^{2} \mathrm{I}_{m_{1} m_{2} m_{3}}^{(3)}[1]\left(p_{x}-p_{z}, p_{y}-p_{z}\right) \\
& +2 p_{z \rho} \mathrm{I}_{m_{1} m_{2} m_{3}}^{(3)}\left[k_{\rho}\right]\left(p_{x}-p_{z}, p_{y}-p_{z}\right)-\hat{\mathrm{T}}_{m_{1} m_{2} m_{3}}^{R}[\square]\left(p_{x}-p_{z}, p_{y}-p_{z}\right) \\
& \hat{\mathrm{Q}}_{m_{1} m_{2} m_{3} m_{4}}^{R}\left[\square \partial_{\mu} \partial_{\nu}\right]=-m_{4}^{2} \mathrm{I}_{m_{1} m_{2} m_{3} m_{4}}^{(4)}\left[k_{\mu} k_{\nu}\right]\left(p_{x}, p_{y}, p_{z}\right)+p_{z \mu} p_{z \nu} \mathrm{I}_{m_{1} m_{2} m_{3}}^{(3)}[1]\left(p_{x}-p_{z}, p_{y}-p_{z}\right) \\
& +p_{z \mu} \mathrm{I}_{m_{1} m_{2} m_{3}}^{(3)}\left[k_{\nu}\right]\left(p_{x}-p_{z}, p_{y}-p_{z}\right)+p_{z \nu} \mathrm{I}_{m_{1} m_{2} m_{3}}^{(3)}\left[k_{\mu}\right]\left(p_{x}-p_{z}, p_{y}-p_{z}\right) \\
& -\hat{\mathrm{T}}_{m_{1} m_{2} m_{3}}^{R}\left[\partial_{\mu} \partial_{\nu}\right]\left(p_{x}-p_{z}, p_{y}-p_{z}\right) \\
& \hat{\mathrm{Q}}_{m_{1} m_{2} m_{3} m_{4}}^{R}\left[\partial_{\mu} \partial_{\nu} \partial_{\rho} \partial_{\sigma}\right]=\mathrm{I}_{m_{1} m_{2} m_{3} m_{4}}^{(4)}\left[k_{\mu} k_{\nu} k_{\rho} k_{\sigma}-\frac{1}{24}\left(\delta_{\mu \nu} \delta_{\rho \sigma}+\delta_{\mu \rho} \delta_{\nu \sigma}+\delta_{\mu \sigma} \delta_{\nu \rho}\right) k^{4}\right]\left(p_{x}, p_{y}, p_{z}\right) \\
& +\frac{1}{24}\left(\delta_{\mu \nu} \delta_{\rho \sigma}+\delta_{\mu \rho} \delta_{\nu \sigma}+\delta_{\mu \sigma} \delta_{\nu \rho}\right)\left(\hat{\mathrm{Q}}_{m_{1} m_{2} m_{3} m_{4}}^{R}[\square \square]\left(p_{x}, p_{y}, p_{z}\right)+\frac{5}{96 \pi^{2}}\right)
\end{aligned}
$$

Table 7: Fourier transforms of three- and four-point renormalized basic functions. $p_{x}$, $p_{y}$ and $p_{z}$ are the conjugate momenta of the coordinate variables $x, y$ and $z$. The momentum-space basic functions on the l.h.s. are assumed to depend on $p_{x}, p_{y}$ (for $\mathrm{T}$ functions) and $p_{x}, p_{y}, p_{z}$ (for $\mathrm{Q}$ functions). The integrals $\mathrm{I}^{(n)}$ are defined in the text. The momentum $k$ is the integration variable. 


\section{References}

[1] D.Z. Freedman, K. Johnson and J.I. Latorre, Nucl. Phys. B371 (1992) 353.

[2] P.E. Haagensen, Mod. Phys. Lett.A7 (1992) 893; R. Muñoz-Tapia, Phys. Lett. B295 (1992) 95; D.Z. Freedman, G. Grignani, K. Johnson and N. Rius, Ann. Phys. 218 (1992) 75; P.E. Haagensen and J.I. Latorre, Ann. Phys. 221 (1993) 77; C. Manuel, Int. J. Mod. Phys. A8 (1993) 3223; D.Z. Freedman, G. Lozano and N. Rius, Phys. Rev. D49 (1994) 1054; J. Comellas, P.E. Haagensen and J.I. Latorre, Int. J. Mod. Phys. A10 (1995) 2819; M. Chaichian, W.F. Chen and H.C. Lee, Phys. Lett. B409 (1997) 325; V.A. Smirnov, Int. J. Mod. Phys. A12 (1997) 4241; D. Anselmi, D.Z. Freedman, M.T. Grisaru, A.A. Johansen, BRX-TH-420, CPTHS.553.0897, HUTP-97/A037, MIT-CTP-2666, hep-th/9708042.

[3] J.I. Latorre, C. Manuel and X. Vilasis-Cardona, Ann. Phys. 231 (1994) 149; G. Dunne and N. Rius, Phys. Lett. B293 (1992) 367; V.A. Smirnov, Theor. Math. Phys. 96 (1993) 974; V.A. Smirnov, Nucl. Phys. B427 (1994) 325; V.A. Smirnov, Z. Phys. C67 (1995) 531; V.A. Smirnov, Theor. Math. Phys. 108 (1997) 953; O. Schnetz, J. Math. Phys. 38 (1997) 738; D. Prange, DESY-97-211, hep-th/9710225.

[4] D.Z. Freedman, K. Johnson, R. Muñoz-Tapia and X. Vilasis-Cardona, Nucl. Phys. B395 (1993) 454.

[5] P.E. Haagensen and J.I. Latorre, Phys. Lett. B283 (1992) 293.

[6] F. del Aguila, A. Culatti, R. Muñoz Tapia and M. Pérez-Victoria, Phys. Lett. B419 (1998) 263.

[7] F. del Aguila, A. Culatti, R. Muñoz Tapia and M. Pérez-Victoria, Nucl. Phys. B504 (1997) 532.

[8] F. del Aguila, A. Culatti, R. Muñoz Tapia and M. Pérez-Victoria, International Workshop on Quantum Effects in MSSM, Universitat Autònoma de Barcelona, September 1997, hep-ph/9711474.

[9] M. Pérez-Victoria, UG-FT-89/98.

[10] T. Hahn and M. Pérez-Victoria, UG-FT-87/98, KA-TP-7-1998.

[11] J. Schwinger, Phys. Rev. 82 (1951) 918; C.S. Lam, Nuovo Cimento 38 (1965) 1754 .

[12] J.H. Lowenstein, Phys. Rev. D4 (1971) 2281; J.H. Lowenstein, Commun. Math. Phys. 24 (1971) 1; Y.M.P. Lam, Phys. Rev. D6 (1972) 2145; Y.M.P. Lam, Phys. Rev. D7 (1973) 2943; T.E. Clark and J.H. Lowenstein, Nucl. Phys. B113 (1976) 109.

[13] O. Piguet, S.P. Sorella, Algebraic Renormalization: Perturbative Renormalization, Symmetries and Anomalies, Berlin (1995), Springer. 
[14] G. 't Hooft and M. Veltman, Nucl. Phys. B44 (1972) 189; C.G. Bollini and J. Giambiagi, Nuovo Cim. 12 B (1972) 20; J.F. Ashmore, Nuovo Cim. Lett. 4 (1972) 289; G.M. Cicuta and E. Montaldi, Nuovo Cim. Lett. 4 (1972) 329.

[15] P. Breitenlohner and D. Maison, Commun. Math. Phys. 52 (1977) 11,39,55.

[16] G. 't Hooft and M. Veltman, Diagrammar, CERN 73-9 (1973).

[17] F. del Aguila and M. Pérez-Victoria, Acta Phys. Polon. B28 (1997) 2279.

[18] M. Abramowitz and I.A. Stegun, Handbook of Mathematical Functions, New York (1972), Dover.

[19] G.J. van Oldenborgh and J.A.M. Vermaseren, Z. Phys. C46 (1990) 425. 